\title{
Galactic cosmic rays on extrasolar Earth-like planets
}

\section{Atmospheric implications}

\author{
J.-M. Grießmeier ${ }^{1,2}$, F. Tabataba-Vakili ${ }^{3}$, A. Stadelmann ${ }^{4}$, J. L. Grenfell ${ }^{5,6}$, and D. Atri ${ }^{7}$ \\ ${ }^{1}$ LPC2E - Université d'Orléans/CNRS, 3A, avenue de la Recherche Scientifique, 45071 Orléans Cedex 2, France \\ e-mail: jean-mathias.griessmeier@cnrs-orleans.fr \\ 2 Station de Radioastronomie de Nançay, Observatoire de Paris - CNRS/INSU, USR 704 - Université Orléans, OSUC, \\ route de Souesmes, 18330 Nançay, France \\ 3 Atmospheric, Oceanic and Planetary Physics, Department of Physics, University of Oxford, Clarendon Laboratory, Parks Road, \\ Oxford OX1 3PU, UK \\ 4 Technische Universität Braunschweig, Mendelssohnstr. 3, 38106 Braunschweig, Germany \\ 5 Zentrum für Astronomie und Astrophysik (ZAA), Technische Universität Berlin (TUB), Hardenbergstr. 36, \\ 10623 Berlin, Germany \\ ${ }^{6}$ Now at: Extrasolare Planeten und Atmosphären (EPA), Institut für Planetenforschung, \\ Deutsches Zentrum für Luft- und Raumfahrt (DLR), Rutherfordstr. 2, 12489 Berlin, Germany \\ 7 Blue Marble Space Institute of Science, 1200 Westlake Ave N Suite 1006, Seattle, WA 98109, USA
}

Received 2 December 2014 / Accepted 16 November 2015

\begin{abstract}
Context. Theoretical arguments indicate that close-in terrestial exoplanets may have weak magnetic fields. As described in the companion article (Paper I), a weak magnetic field results in a high flux of galactic cosmic rays to the top of the planetary atmosphere. Aims. We investigate effects that may result from a high flux of galactic cosmic rays both throughout the atmosphere and at the planetary surface.

Methods. Using an air shower approach, we calculate how the atmospheric chemistry and temperature change under the influence of galactic cosmic rays for Earth-like $\left(\mathrm{N}_{2}-\mathrm{O}_{2}\right.$ dominated) atmospheres. We evaluate the production and destruction rate of atmospheric biosignature molecules. We derive planetary emission and transmission spectra to study the influence of galactic cosmic rays on biosignature detectability. We then calculate the resulting surface UV flux, the surface particle flux, and the associated equivalent biological dose rates.

Results. We find that up to $20 \%$ of stratospheric ozone is destroyed by cosmic-ray protons. The effect on the planetary spectra, however, is negligible. The reduction of the planetary ozone layer leads to an increase in the weighted surface UV flux by two orders of magnitude under stellar UV flare conditions. The resulting biological effective dose rate is, however, too low to strongly affect surface life. We also examine the surface particle flux: For a planet with a terrestrial atmosphere (with a surface pressure of $1033 \mathrm{hPa}$ ), a reduction of the magnetic shielding efficiency can increase the biological radiation dose rate by a factor of two, which is non-critical for biological systems. For a planet with a weaker atmosphere (with a surface pressure of $97.8 \mathrm{hPa}$ ), the planetary magnetic field has a much stronger influence on the biological radiation dose, changing it by up to two orders of magnitude.

Conclusions. For a planet with an Earth-like atmospheric pressure, weak or absent magnetospheric shielding against galactic cosmic rays has little effect on the planet. It has a modest effect on atmospheric ozone, a weak effect on the atmospheric spectra, and a noncritical effect on biological dose rates. For planets with a thin atmosphere, however, magnetospheric shielding controls the surface radiation dose and can prevent it from increasing to several hundred times the background level.
\end{abstract}

Key words. planets and satellites: terrestrial planets - planets and satellites: magnetic fields - planets and satellites: atmospheres cosmic rays - astrobiology

\section{Introduction}

The number of known extrasolar planets is steadily growing, as is the number of known Earth-like and - Super-Earth - like planets (i.e. planets with a mass $M \leq 10 M_{\oplus}$ ) around M-dwarf stars. Recent estimations based on the Kepler Input Catalog indicate that the occurrence rate of planets with a radius $0.5 R_{\oplus} \leq R \leq$ $4 R_{\oplus}$ orbiting an $\mathrm{M}$ dwarf in less than 50 days is $0.9_{-0.03}^{+0.04}$ planets per star (Dressing \& Charbonneau 2013). A considerable number of these planets $(\sim 15$ to $50 \%)$ could be located in the socalled liquid water habitable zone of their host star.

However, the classical definition of the habitable zone (e.g., Kasting et al. 1993; Selsis et al. 2007) is based solely on the potential of having liquid water on the planetary surface (hence the more precise name of liquid water habitable zone). Clearly, a number of additional factors can also play an important role for habitability (see e.g., Lammer et al. 2009, 2010).

One of these additional conditions probably is the presence of a planetary magnetic field. Magnetic fields on super-Earths around M-dwarf stars are likely to be weak and short-lived in the best case or even non-existent in the worst case. The relevance of such fields and their potential detectability is discussed elsewhere (Grießmeier 2014). Here, we look at one habitabilityrelated consequence of a weak planetary magnetic field, namely the enhanced flux of galactic cosmic-ray (GCR) particles to the planetary atmosphere, with potential implications ranging from changes in the atmospheric chemistry to an increase of the radiation dose on the planetary surface. 
The effects of GCRs on the planetary atmospheric chemistry were calculated by Grenfell et al. (2007a) for $\mathrm{N}_{2}-\mathrm{O}_{2}$ atmospheres. They found that for an unmagnetized planet, GCRs can reduce the total ozone column by almost $20 \%$, which is not sufficent to strongly influence the biomarker signature. Thus, they concluded that biomarkers are robust against GCRs for the scenarios studied.

GCRs also lead to a flux of secondary particles which can reach the planetary surface. The resulting surface radiation dose was evaluated by Atri et al. (2013). For an Earth-like atmosphere with a surface pressure of $1033 \mathrm{hPa}$, they find that the absence of magnetospheric shielding can increase the surface biological dose rate by up to a factor of $\sim 2$. They also indicate that atmospheric shielding dominates over magnetospheric shielding; compared to a thin atmosphere (10 times less dense than on Earth), an atmosphere of $1033 \mathrm{hPa}$ reduces the dose rate by almost 3 orders of magnitude.

The exact severity of these effects, however, depends on the particle energy range considered, and on the intrinsic planetary magnetic field strength. For planets with a strong magnetic field, most galactic cosmic-ray particles are deflected, whereas for weakly magnetized planets, the majority of the particles can reach the planetary atmosphere. In previous work (Grießmeier et al. 2005, 2009), the flux of galactic cosmic rays to the atmosphere of extrasolar planets has been evaluated on the basis of a simple estimate for the planetary magnetic moment. However, such quantitative estimates of magnetic fields can be over-simplistic. More complex approaches, however, yield values which are not only model-dependent, but also depend on the precise planetary parameters. For this reason, we have re-evaluated the cosmic-ray flux in the companion article (Grießmeier et al. 2015, hereafter "Paper I"), including primary particles over a wider energy range. More importantly, we now take a more general approach concerning the planetary magnetic moment: Instead of applying a model for the planetary magnetic moment, we showed how magnetic protection varies as a function of the planetary magnetic dipole moment, in the range $0.0 \mathcal{M}_{\oplus} \leq \mathcal{M} \leq 10.0 \mathcal{M}_{\oplus}$ for the magnetic moment, and in the range of $16 \mathrm{MeV} \leq E \leq 524 \mathrm{GeV}$ for the particle energy.

The aim of the current study is to use this greatly expanded parameter range and repeat the analysis of earlier studies (Grenfell et al. 2007a; Atri et al. 2013). In addition to the new cosmic-ray fluxes from Paper I, the main differences with respect to the approach of previous work (Grenfell et al. 2007a; Atri et al. 2013) are the following:

- The climate-chemistry atmospheric model has been updated, as explained in Sect. 3.1.

- The calculation of the photochemical response to cosmic rays has been updated, as summarized in Sect. 3.1 (see Tabataba-Vakili et al. 2016, for details).

- We have added the analysis of planetary transmission and emission spectra (Sect. 4.2).

- We have added the analysis of surface UV flux, and present results for UV-A, UV-B, and biologically weighted UV flux on the planetary surface (Sect. 4.3).

This paper is organized as follows (see also Fig. 1): in Sect. 2, we present the planetary parameters used in our calculations. Sect. 3 decribes the models and numerical tools we use: The atmospheric chemistry model is discussed in Sect. 3.1 and the surface particle flux and radiation dose calculation is presented in Sect. 3.2. The galactic cosmic-ray fluxes are computed in Paper I. We discuss the implications of these fluxes in Sect. 4, i.e. the modification of the atmospheric chemistry (Sect. 4.1), the

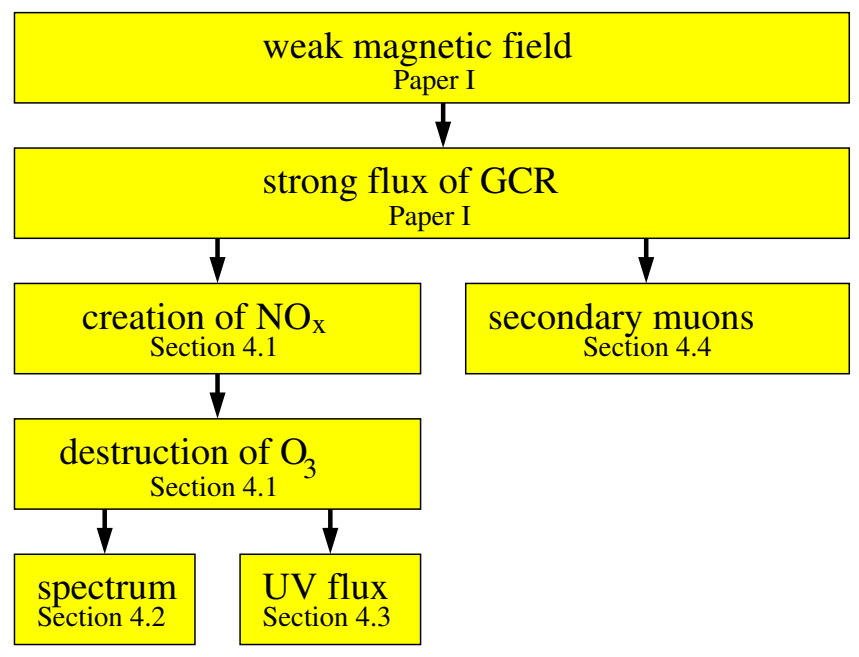

Fig. 1. Effects of galactic cosmic rays discussed in this work and in the companion article (Paper I).

detectability of biosignature molecules (Sect. 4.2), the UV flux at the planetary surface (Sect. 4.3) and the surface radiation dose rate (Sect. 4.4). Section 5 closes with some concluding remarks.

\section{The planetary situation}

In Paper I, we calculated a large number of representative cases. This allows us to study systematically the influence of the planetary magnetic field on the flux of GCRs to the planet. In this way, we explore the range $0.0 \mathcal{M}_{\oplus} \leq \mathcal{M} \leq 10.0 \mathcal{M}_{\oplus}$ for the magnetic moment, and the range of $16 \mathrm{MeV} \leq E \leq 524 \mathrm{GeV}$ for the particle energy.

The following parameters are kept fixed: the stellar mass $M_{\star}$ $\left(M_{\star}=0.45 M_{\odot}\right)$, the stellar radius $R_{\star}\left(R_{\star}=0.41 R_{\odot}\right)$, and the orbital distance $d(d=0.153 \mathrm{AU})$. We are thus looking at the case of a planet in orbit around a star equivalent to AD Leonis (an M4.5 dwarf star). In addition, we keep constant the planetary mass $M_{\mathrm{p}}\left(M_{\mathrm{p}}=1.0 M_{\oplus}\right)$ and the planetary radius $R_{\mathrm{p}}$ $\left(R_{\mathrm{p}}=1.0 R_{\oplus}\right)$. The only planetary or stellar parameter which we varied in the present study is the planetary magnetic dipole moment $\mathcal{M}$. The minimum value of the magnetic dipole moment in this study is 0 , which corresponds to an unmagnetized planet. The maximum value of the magnetic dipole moment we study is 10 times the present Earth value, which corresponds to an extremely strongly magnetized planet.

Already for Earth-mass rocky exoplanets, a variety of atmospheric compositions can be envisaged. Super-Earths may have a very different atmospheric chemistry from Earth. Key processes expected to influence their atmosphere include: the origin of the primary atmosphere, the presence (or absence) of life, the presence (or absence) of a water ocean, atmospheric escape and the importance of outgassing (see, e.g., Hu et al. 2012, 2013; Hu \& Seager 2014). Having a different atmospheric composition obviously has an effect on cosmic-ray transport and influence the atmospheric profiles of the biomarker molecules we study. In this work, we assume an Earth-like atmosphere of $1033 \mathrm{hPa}$ surface pressure with $\mathrm{N}_{2}$ and $\mathrm{O}_{2}$ as the major constituents, and with biogenic gas emissions as on modern Earth. The planetary atmospheric parameters are described in more detail in Sect. 3.1 and in Rauer et al. (2011). 


\section{Models used}

In this section, we describe the models used throughout this article. The stellar wind model, magnetic field model, and cosmicray propagation model are presented in Paper I. The particle fluxes calculated in Paper I are used as input into a coupled climate-chemistry atmospheric column model. This model uses an air shower approach to estimate GCR-induced photochemical effects and the resulting modification of atmospheric chemistry, as described in Sect. 3.1. The cosmic-ray fluxes of Paper I are also used as input for the calculation of the particle flux to the surface. We describe our surface particle flux and radiation dose model in Sect. 3.2. The results obtained with these models are given in Sect. 4.

\subsection{Climate-chemistry atmospheric column model}

To study the response of the atmosphere to the cosmic-ray flux, we use a Coupled Climate-Chemistry Atmospheric Column Model. The details of this model are decribed elsewhere (Rauer et al. 2011; Grenfell et al. 2012, 2013, and references therein). Since Grenfell et al. (2007a) we include a new offline binning routine for the input stellar spectra and a variable vertical atmospheric height (see Rauer et al. 2011). The code has two main modules, namely, a radiative-convective climate module (Sect. 3.1.1) and a chemistry module (Sect. 3.1.2). The photochemical response induced by cosmic rays is modeled according to Grenfell et al. (2007a), including some model updates described in Tabataba-Vakili et al. (2016) (Sect. 3.1.3). Finally, the output of the atmospheric model is fed into a theoretical spectral model (Sect. 3.1.4).

The modification of atmospheric chemistry by galactic cosmic rays for our configurations are described in Sects. 4.1-4.3.

\subsubsection{Climate module}

The Climate Module is a global-average, stationary, hydrostatic atmospheric column model ranging from the surface up to altitudes with a pressure of $6.6 \times 10^{-5}$ bar (for the modern Earth this corresponds to a height of $\sim 70 \mathrm{~km}$ ). Starting values of composition, pressure, and temperature are based on modern Earth. The radiative transfer is based on the work of Toon et al. (1989) for the shortwave region, and on the RRTM (Rapid Radiative Transfer Module, Mlawer et al. 1997) for thermal radiation. This uses 16 spectral bands by applying the correlated k-method for major absorbers. Its validity range (see Mlawer et al. 1997) for a given height corresponds to Earth's modern mean temperature $\pm 30 \mathrm{~K}$ for pressures between $10^{-5}$ and 1.05 bar and for a $\mathrm{CO}_{2}$ abundance from modern up to 100 times modern. The shortwave radiation scheme features 38 spectral intervals for the main absorbers, including Rayleigh scattering for $\mathrm{N}_{2}, \mathrm{O}_{2}$, and $\mathrm{CO}_{2}$ with cross-sections based on Vardavas \& Carver (1984). The climate scheme uses a constant, geometrical-mean, solarzenith angle of $60^{\circ}$. In the troposphere, lapse rates are derived assuming moist adiabatic convection and using the Schwarzschild criterion. Tropospheric humidity comes from Earth observations (Manabe \& Wetherald 1967).

For the reference case of the Sun, we employed a high resolution solar spectrum based on Gueymard (2004) binned to the wavelength intervals employed in the photochemistry and climate schemes of the column model.

For the standard M dwarf scenario (the "chromospherically active" case of Sect. 4.3), we assume a stellar spectrum identical to that of AD Leonis (an M4.5 dwarf star). The spectrum is derived from observations of the IUE satellite and photometry in the visible (Pettersen \& Hawley 1989), using observations in the near IR (Leggett et al. 1996) and based on a nextGen stellar model spectrum for wavelengths beyond 2.4 microns (Hauschildt et al. 1999).

We also study scenarios with stellar UV flares ("long flare" and "short flare" scenarios). In those cases, the stellar UV spectrum was taken from Segura et al. (2010, Fig. 3, bold blue line, scaled for distance). These cases are described in detail in Sect. 4.3.

Clouds are not included directly, although they are considered in a straightforward manner by adjusting surface albedo to achieve a mean surface temperature of the modern Earth (288 K).

After convergence, the climate module outputs the temperature, water abundance and pressure. These variables are interpolated from the climate grid (52 levels) onto the chemistry grid (64 levels) (both grids extend from the surface up to about the mid-mesosphere) and are then used as start values for the chemistry module. This, in turn runs to convergence and then outputs and interpolates the concentrations of key radiative species $\mathrm{CH}_{4}$, $\mathrm{H}_{2} \mathrm{O}, \mathrm{O}_{3}$ and $\mathrm{N}_{2} \mathrm{O}$ to be used as start values for the next cycle of the climate module. This process is repeated back and forth between the climate and chemistry modules until overall convergence is reached.

\subsubsection{Chemistry module}

The Chemistry Module has been detailed in Pavlov \& Kasting (2002). Our stationary scheme has 55 species for more than 200 reactions with chemical kinetic data taken from the Jet Propulsion Laboratory (JPL) Report (Sander et al. 2003). Molecules are photolyzed in 108 spectral intervals from 175.4-855 $\mathrm{nm}$ with an additional nine intervals from 133-173 nm and a tenth interval in the Lyman-alpha. The original chemistry scheme is described in Kasting et al. $(1984 a, b)^{1}$. For the absorption cross-sections of key species undergoing photolysis, NO is based on Cieslik \& Nicolet (1973), $\mathrm{N}_{2} \mathrm{O}_{5}$ on Yao et al. (1982), $\mathrm{NO}_{2}$ on Jones \& Bayes (1973), $\mathrm{O}_{3}$ was taken from Malicet et al. (1995) and Moortgat \& Kudszus (1978), and $\mathrm{NO}_{3}$ was taken from Magnotta \& Johnston (1980).

We assume a planet with an Earth-like development, i.e. $\mathrm{N}_{2}-$ $\mathrm{O}_{2}$ dominated atmosphere, a modern Earth biomass, etc. The scheme reproduces modern Earth's atmospheric composition with a focus on biosignature molecules (e.g., $\mathrm{O}_{3}, \mathrm{~N}_{2} \mathrm{O}$ ) and major greenhouse gases such as $\mathrm{CH}_{4}$. The module calculates the converged solution of the standard 1D continuity equations using an implicit Euler scheme. Mixing occurs via Eddy diffusion coefficients $(\mathrm{K})$ based on Earth observations (Massie \& Hunten 1981). Constant surface biogenic (e.g., $\mathrm{CH}_{3} \mathrm{Cl}, \mathrm{N}_{2} \mathrm{O}$ ) and source gas (e.g., $\mathrm{CH}_{4}, \mathrm{CO}$ ) emissions were employed based on the modern Earth (see Grenfell et al. 2011, for more details). $\mathrm{H}_{2}$ was removed at the surface as detailed in Rauer et al. (2011). Also calculated are modern-day tropospheric lightning emissions of nitrogen monoxide (NO), volcanic sulphur emissions of $\mathrm{SO}_{2}$ and $\mathrm{H}_{2} \mathrm{~S}$, and a constant downward flux of $\mathrm{CO}$ and $\mathrm{O}$ at the upper boundary, which represents the photolysis products of $\mathrm{CO}_{2}$. Dry and wet deposition is included for long-lived species via

1 Further information can also be found at: http://vpl.astro. washington.edu/sci/AntiModels/models09.html where the original source code is available. 
deposition velocities (for dry deposition) and Henry's law constants (for wet deposition).

\subsubsection{Cosmic-ray scheme}

For the cosmic-ray scheme, we use an air shower approach based on Grenfell et al. (2007a, 2012) and Tabataba-Vakili et al. (2016). The top of atmosphere (TOA) time-average proton fluxes from the magnetospheric cosmic-ray model (Paper I) are input into the chemistry module at the upper boundary. Secondary particles are generated, which leads to $\mathrm{NO}_{x}$ production. In the present work our scheme was updated to produce 1.25 odd nitrogen atoms per ion pair produced by cosmic rays, according to Jackman et al. (1980), based on calculations of dissociation branching ratios from relativistic particle impact cross sections (Porter et al. 1976). We introduced a parameterization whereby the GCR-induced N-production was split into two channels, i.e $45 \%$ ground-state $\mathrm{N}$ and $55 \%$ excited-state $\mathrm{N}$ (see Jackman et al. 2005 , and references therein). We also introduced an energydependence to the total $\mathrm{N}_{2}$ ionization cross section by electron impact (Tabataba-Vakili et al. 2016), replacing the constant electron impact cross section of $1.75 \times 10^{-16} \mathrm{~cm}^{2}$ previously used with the energy-dependent cross section of Itikawa (2006). Additionally, the input parameters for the Gaisser-Hillas formula were extended up to $524 \mathrm{GeV}$ to be consistent with the cosmic-ray calculation. Finally, in the Gaisser-Hillas scheme the parametrization was changed. The parameter of the proton attenuation length $\left(80 \mathrm{~g} / \mathrm{cm}^{2}\right)$ was replaced with a depth of first interaction of $5 \mathrm{~g} / \mathrm{cm}^{2}$ according to Alvarez-Muñiz et al. (2002), which led to a closer match with observations. For more details of the above updates and their effects see Tabataba-Vakili et al. (2016). Note that the cosmic-ray scheme in the current work includes production of only nitrogen oxides in the photochemistry (whereas in a newly-developed version as described by Tabataba-Vakili et al. (2016) the cosmic rays lead to the production of both nitrogen oxides and hydrogen oxides).

\subsubsection{Theoretical spectral model}

To calculate the spectral appearance of our model atmospheres, we use the SQuIRRL code (Schwarzschild Quadrature InfraRed Radiation Line-by-line, Schreier \& Schimpf 2001). This code was designed to model radiative transfer with a high resolution in the IR region for a spherically symmetric atmosphere (taking arbitrary observation geometry, instrumental field of view, and spectral response function into account). The scheme assumes local thermodynamic equilibrium; for each layer, a Planck function is used to determine the emission. Cloud and haze free conditions without scattering are assumed. Absorption coefficients are calculated using molecular line parameters from the HITRAN 2008 database (Rothman et al. 2009), and emission spectra are calculated assuming a pencil beam at a viewing angle of $38^{\circ}$ as used, for example, in Segura et al. (2003). SQuIRRL has been validated e.g. by Melsheimer et al. (2005).

\subsection{Surface particle flux and radiation dose calculation}

If particles from a cosmic-ray shower reach the planetary surface, biological systems on the planetary surface can be strongly influenced and even damaged by this secondary radiation. In order to assess the expected biological damage, we simulate the air shower and its passage through the atmosphere. In the case of Earth, muons contribute $75 \%$ of the equivalent dose rate at the surface (O'Brien et al. 1996), so that our focus lies on these particles, but the contribution of neutrons and electrons are included, too.

Cosmic ray propagation in the atmosphere is a challenging problem, beyond the scope of analytical tools because one has to compute a variety of hadronic and electromagnetic interactions occurring in the atmosphere. Therefore, as a complement to the cosmic-ray air shower model which we use for secondary electrons (see Sect. 3.1.3), we also use a robust Monte Carlo package, CORSIKA v.6990 (Heck et al. 1998, 2012), which is widely used to simulate air showers for major particle detection experiments. The code makes use of a number of packages to model high and low energy hadronic interaction processes and all electromagnetic interactions of charged particles. We take the input cosmic-ray spectrum calculated for different magnetic field cases (the output of Paper I) and model particle propagation with 20 million primary particles for each case. Using such a large ensemble of particles is necessary to reduce the numerical error as much as possible. Hadronic interactions up to $80 \mathrm{GeV}$ were modeled using the GHEISHA model and above $80 \mathrm{GeV}$ using the SIBYLL 2.1 high-energy hadronic interaction model. None of the "thinning" options were used so that no particle information was lost. The final output gives the momentum and types of particles hitting the ground for 20 million primaries. Primaries are incident at the top of the atmosphere from random angles with energies falling randomly according to the energy spectrum. The electromagnetic interactions enhance the atmospheric ionization rate and change the atmospheric chemistry (Atri et al. 2010). For energies of the primary particles above $8 \mathrm{GeV}$, hadronic interactions produce particles (such as muons and neutrons), some of which reach the ground and contribute to the radiation dose (Atri et al. 2011, 2013).

The surface radiation calculation thus is similar to the calculations of Atri et al. (2013). However, we extend the primary particle energy range and increase the range of planetary magnetic fields. We use an Earth-like atmospheric composition, but compare two different values of atmospheric depth: $1036 \mathrm{~g} / \mathrm{cm}^{2}$ (equivalent to a surface pressure of $1033 \mathrm{hPa}$ ), and $100 \mathrm{~g} / \mathrm{cm}^{2}$ (equivalent to a surface pressure of $97.8 \mathrm{hPa}$ ). The resulting surface radiation equivalent dose rate is described in Sect. 4.4.

\section{Implications}

The interaction of GCR particles with a planet and its atmosphere can lead to a host of interesting effects, several of which have been suggested to be potentially relevant for habitability. The excellent review by Dartnell (2011) mentions effects as diverse as: the modification of the atmospheric chemistry (which we discuss below), the excitation and ionization of atomic and molecular species, the creation of an ionosphere, ions driving atmospheric chemistry and potentially weather and climate dynamics, the possible influence on atmospheric lightning, the production of organic molecules within the atmosphere, the destruction of stratospheric ozone (see below), the possible sterilization of the planetary surface (see below), and the degradation of biosignatures (see below).

In the present work, we focus on the following effects: Based on the atmospheric model of Sect. 3.1, we look at the modification of the atmospheric chemistry (Sect. 4.1), verify the stability of biosignature molecules against destruction by cosmic rays (Sect. 4.2), and analyze the enhanced UV flux resulting from a weakened ozone layer (Sect. 4.3). Finally, with the surface radiation calculation of Sect. 3.2, we study the biological radiation dose at the planetary surface (Sect. 4.4). 

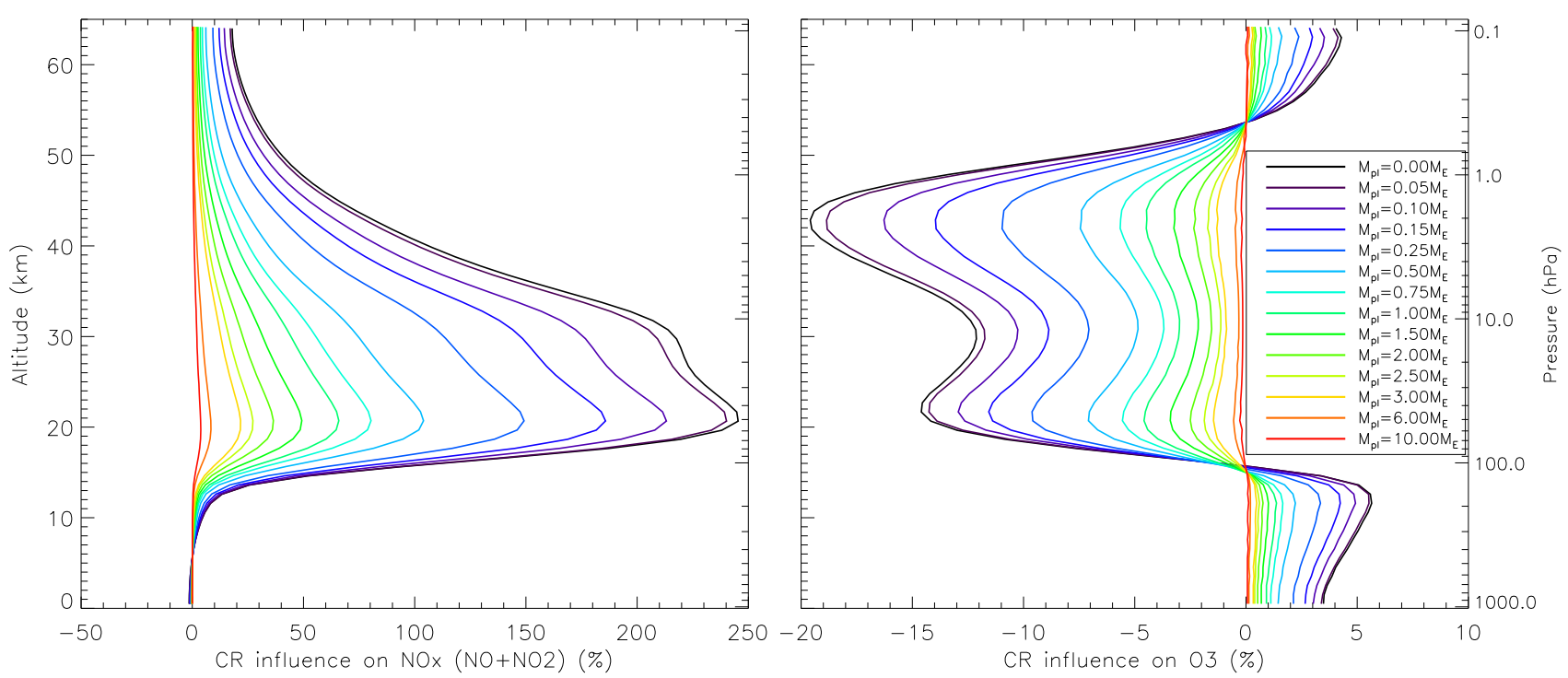

Fig. 2. Altitude-dependent change of the volume mixing ratio of $\mathrm{NO}_{x}$ (i.e. $\left.\mathrm{NO}+\mathrm{NO}_{2}\right)($ left $)$ and $\mathrm{O}_{3}$ (right) for exoplanets with a magnetic moment of $\mathcal{M}=0.0,0.05,0.1,0.15,0.25,0.5,0.75,1.0,1.5,2.0,2.5,3.0,6.0$ and $10.0 \mathcal{M}_{\oplus}$, relative to a case without cosmic rays.

\subsection{Modification of atmospheric chemistry}

After having traversed the planetary magnetosphere, the galactic cosmic-ray protons reach the planetary atmosphere. On the way through the atmosphere, they interact with neutral gas particles, and create secondary electrons via impact ionization:

$\mathrm{p}^{+}+\mathrm{X} \rightarrow \mathrm{p}^{+}+\mathrm{X}^{+}+\mathrm{e}^{-}$.

These free electrons break $\mathrm{N}_{2}$ molecules, which leads to the formation of $\mathrm{NO}_{x}$ :

$\mathrm{N}_{2}+\mathrm{e}^{-} \rightarrow 2 \mathrm{~N}+\mathrm{e}^{-}$

$\mathrm{N}+\mathrm{O}_{2} \rightarrow \mathrm{NO}+\mathrm{O}$.

Depending on local conditions and the dominating ozone production mechanism, these NO molecules can either destroy ozone (if ozone was created by the Chapman mechanism), or may create ozone (if ozone is formed via a smog mechanism). While both mechanisms are included in our model, ozone production by a Chapman mechanism dominates in the case of a planet orbiting a chromospherically active M-dwarf star, especially at altitudes above $20 \mathrm{~km}$ (Grenfell et al. 2013), so that in our case NO mostly destroys stratospheric ozone by catalytic cycles. Their results suggest that for planets orbiting chromospherically active M-dwarf stars, this process is dominant over most other pathways of ozone destruction, which are also included in our model (or ozone loss via $\mathrm{CO}$ oxidation, see Grenfell et al. 2013). The case of catalytic $\mathrm{HO}_{x}$ created by cosmic rays is investigated in more detail elsewhere (Tabataba-Vakili et al. 2016). Thus, we have (Crutzen 1970):

$\mathrm{NO}+\mathrm{O}_{3} \rightarrow \mathrm{NO}_{2}+\mathrm{O}_{2}$

$\mathrm{NO}_{2}+\mathrm{O} \rightarrow \mathrm{NO}+\mathrm{O}_{2}$

net: $\mathrm{O}+\mathrm{O}_{3} \rightarrow 2 \mathrm{O}_{2}$.

The $\mathrm{O}$ atom in Eq. (5) is created e.g. by photolysis of $\mathrm{O}_{2}$ by photons in the Herzberg region of $\sim 180 \mathrm{~nm}$.

Equation (6) describes the net reaction of the catalytic cycle: Ozone molecules are transformed into molecular oxygen. $\mathrm{NO}_{x}$ is regenerated, so that a single molecule can contribute to the destruction of a large number of ozone molecules.
In order to quantify the effects described by Eqs. (1) to (6) for extrasolar planets around M-dwarf stars, we use the coupled climate-chemistry model described in Sect. 3.1. In the following, we present the results obtained with this model, probing planets with magnetic moments in the range $0.0 \mathcal{M}_{\oplus} \leq \mathcal{M} \leq 10.0 \mathcal{M}_{\oplus}$.

Figure 2 shows how the influx of galactic cosmic rays into the planetary atmosphere changes the atmospheric compositional profile. The left panel of the figure shows the altitudedependent relative change in the $\mathrm{NO}_{x}$ volume mixing ratio. In the case of a weak magnetic field, the increased influx of galactic cosmic rays enhanced the $\mathrm{NO}_{x}$ by up to a factor 3.5. This enhancement peaks in the lower stratosphere, where the air shower interaction is strong (e.g. Tabataba-Vakili et al. 2016). The right panel of Fig. 2 shows that the increased $\mathrm{NO}_{x}$ catalytically destroys up to $20 \%$ of stratospheric $\mathrm{O}_{3}$. In the troposphere, however, $\mathrm{O}_{3}$ abundances increase by up to $6 \%$ because $\mathrm{NO}_{x}$ stimulates the smog mechanism.

Figure 3 shows the effect of GCRs on $\mathrm{CH}_{4}$ and $\mathrm{H}_{2} \mathrm{O}$. Both chemical species decrease in concentration with decreasing magnetospheric protection (i.e. increasing GCR flux). The left panel shows that the $\mathrm{CH}_{4}$ abundance decreases as shielding decreases. This is explained by the fact that the increased amount of cosmic-ray particles leads to a higher $\mathrm{NO}_{x}$ abundance, which in turn leads to more $\mathrm{OH}$ :

$\mathrm{NO}+\mathrm{HO}_{2} \rightarrow \mathrm{NO}_{2}+\mathrm{OH}$.

The increased $\mathrm{OH}$ abundance leads to a more efficent destruction of $\mathrm{CH}_{4}$ :

$\mathrm{CH}_{4}+\mathrm{OH} \rightarrow \mathrm{CH}_{3}+\mathrm{H}_{2} \mathrm{O}$.

Thus, less shielding (i.e. more cosmic rays) leads to less $\mathrm{CH}_{4}$. A detailed analysis of the runs in the current paper suggests that $\mathrm{OH}$ is the dominant in-situ sink: other sinks e.g. due to reaction with excited oxygen atoms or with atomic chlorine are weaker and have $\mathrm{CH}_{4}$ removal rates which are lower by at least two orders of magnitude. When we increase the planetary magnetic moment from 0 to 10 times the Earth's value, the atmospheric $\mathrm{CH}_{4}$ increased modestly by $7 \%$.

The right panel of Fig. 3 shows the effect of galactic cosmic rays on the $\mathrm{H}_{2} \mathrm{O}$ abundance, which decreases as the magnetic 

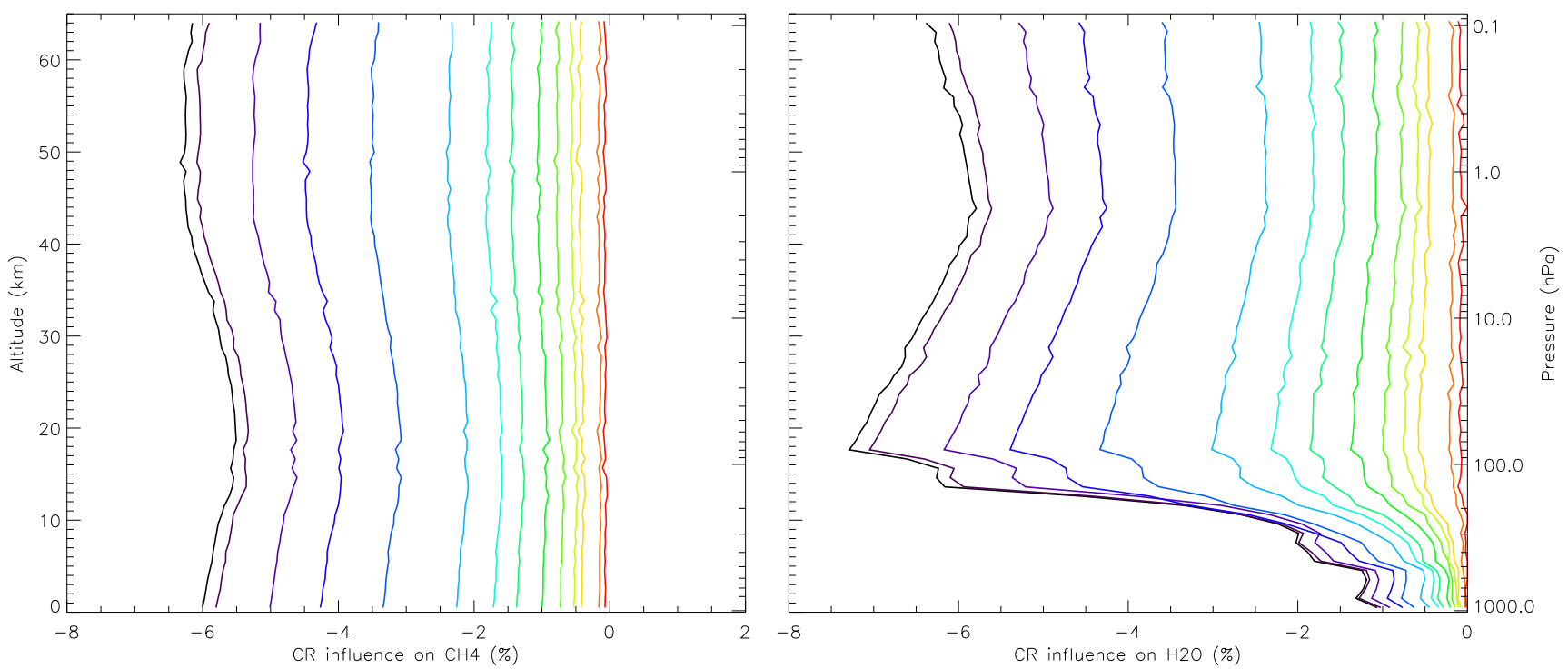

Fig. 3. As Fig. 2 but for $\mathrm{CH}_{4}$ (left) and $\mathrm{H}_{2} \mathrm{O}($ right).
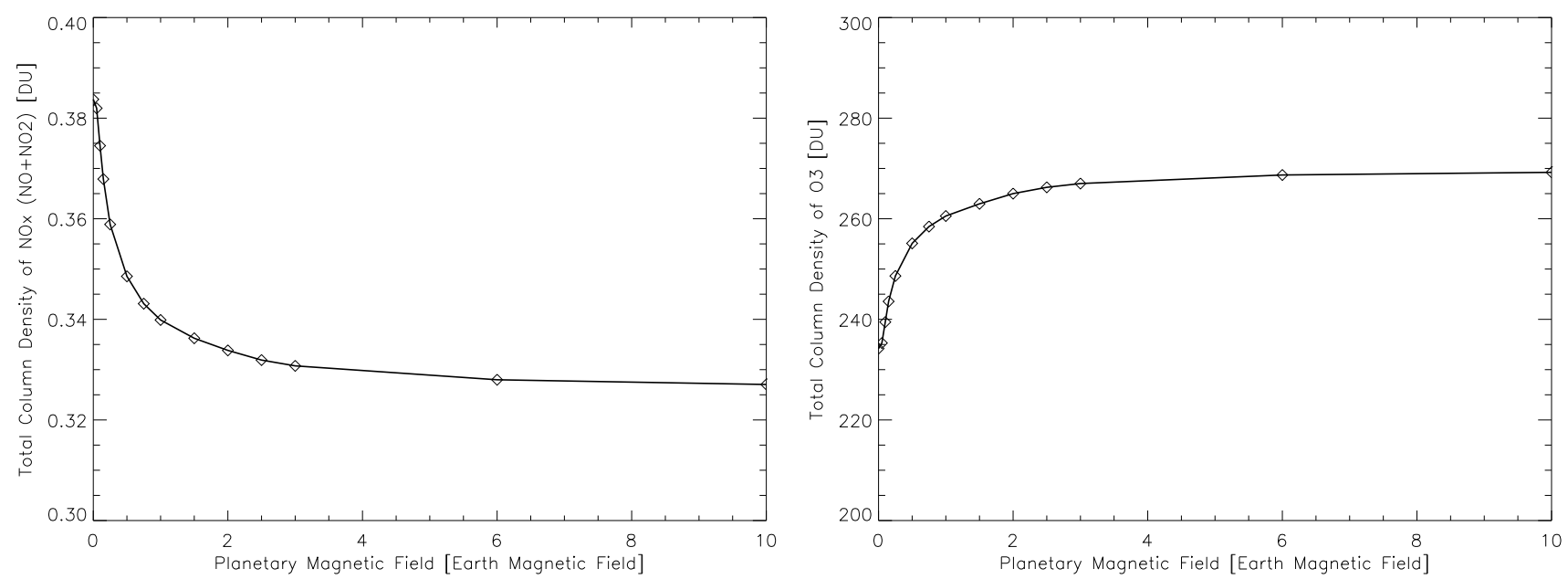

Fig. 4. Column density of $\mathrm{NO}_{x}$ (i.e. $\mathrm{NO}+\mathrm{NO}_{2}$, left panel) and $\mathrm{O}_{3}$ (right panel) as a function of exoplanetary magnetic moment. One Dobson Unit (DU) is equivalent to a column density of $2.69 \times 10^{16}$ molecules $\mathrm{cm}^{-2}$.

shielding decreases (i.e. cosmic rays increase). This is a direct effect of the decrease in $\mathrm{CH}_{4}$, see Eq. (8).

Figure 4 shows how the influx of galactic cosmic rays changes the total atmospheric column density for $\mathrm{NO}_{x}$ and $\mathrm{O}_{3}$. One can see that under the influence of galactic cosmic rays, the $\mathrm{NO}_{x}$ column varies by up to $15 \%$, while the $\mathrm{O}_{3}$ column varies by up to $13 \%$.

If one compares the $0.15 \mathcal{M}_{\oplus}$ case of the present study with the equivalent (run 3) of our previous work using an earlier model version (Grenfell et al. 2007a), one finds that the results are in good agreement, despite the widened proton energy range and the numerous updates to the routines of the climatechemistry atmospheric model (see Sect. 3.1 for details). For example, run 3 of Grenfell et al. (2007a) found a 10\% ozone decrease at $30 \mathrm{~km}$ altitude, and an ozone column value decreasing by $16 \%$, compared to the case without GCR (their run 2 ). In our current model $\left(0.15 \mathcal{M}_{\oplus}\right.$ case $)$, we have a $10 \%$ ozone decrease at $30 \mathrm{~km}$ altitude and an ozone column value decreasing by $10 \%$ compared to a case without cosmic rays.

Similarly, for their run 4 (corresponding to the $0.0 \mathcal{M}_{\oplus}$ case here), Grenfell et al. (2007a) found a 12-13\% ozone decrease at
$30 \mathrm{~km}$ altitude, and an ozone column value decreasing by $19 \%$ when compared to the case without GCR (their run 2). In our current model $\left(0.0 \mathcal{M}_{\oplus}\right.$ case $)$, we have a $12 \%$ ozone decrease at $30 \mathrm{~km}$ altitude, and an ozone column value decreasing by $13 \%$. This similarity arises e.g. from constraints in the maximum NO production rate by measurement data for the Earth reference case (Tabataba-Vakili et al. 2016).

Absolute values of ozone columns for the AD Leonis scenarios are lower in the present work than in earlier modeling versions (e.g. Grenfell et al. 2007a). Note that in the present work we place the planet at the distance from the star where the net incoming energy equals one solar constant (instead of a previous approach where the surface albedo was adjusted in order to reach a surface temperature of $288.0 \mathrm{~K}$ ). Other recent model updates are described in Rauer et al. (2011).

In the present work there is a clear smog signal (e.g. Fig. 2, right panel) leading to an increase of tropospheric $\mathrm{O}_{3}$ when $\mathrm{NO}_{x}$ increases. This effect was not so evident in earlier studies (e.g. Grenfell et al. 2007a). On Earth, smog ozone production has different regimes where it can be sensitive to a) changes in organic species (e.g., $\mathrm{CH}_{4}, \mathrm{CO}$ etc.) or b) to changes in $\mathrm{NO}_{x}$. In the 
present work the absolute $\mathrm{CH}_{4}$ is higher than in Grenfell et al. (2007a) (the overall $\mathrm{CH}_{4}$ response is chemically complex, related to changes in $\mathrm{OH}$, see Grenfell et al. (2012) for some discussion). Higher $\mathrm{CH}_{4}$ is consistent with a saturation of the smog mechanism with respect to organic species, hence a more significant role of smog $\mathrm{O}_{3}$ to changes in $\mathrm{NO}_{x}$ - more work however is required to investigate this further.

Similarly to Grenfell et al. (2007a), we reach the conclusion that atmospheric biosignature molecules are not strongly influenced by GCRs for Earth-like planets in the habitable zone around M-dwarf stars. The case is, however, different for solar energetic particles, which can strongly modify the abundance of ozone in the planetary atmosphere (Segura et al. 2010; Grenfell et al. 2012). Using the updated model as above, this case is reevaluated in Tabataba-Vakili et al. (2016).

\subsection{Spectral signature of biosignature molecules}

A particularly useful method to study exoplanets is the analysis of their atmospheric composition via emission or transmission spectroscopy. In the case of Earth-mass exoplanets or super-Earths, a central goal involves the search for molecules which could indicate the presence of life and which cannot be explained by inorganic chemistry alone, so-called "biosignature molecules" (sometimes also called "biomarkers"). Several telescopes that are currently planned or under construction (e.g., E-ELT, JWST) could possibly detect spectral biosignatures on potentially rocky planets around nearby stars, although this remains very challenging.

Clearly, care has to be taken when selecting and interpreting biosignature molecules. Proposed biosignature molecules include oxygen (when produced in large amounts by photosynthesis), ozone (mainly produced from oxygen) and nitrous oxide (produced almost exclusively from bacteria).

It is important to understand all effects which can modify the abundances of these molecules. For example, Grenfell et al. (2007b) look at the response of biosignature chemistry (e.g. ozone) on varying planetary and stellar parameters (orbital distance and stellar type: F, G, and K). Rauer et al. (2011) and Grenfell et al. (2013) study the effect of stellar spectral type (from M0 to M7, plus the case of the active M-dwarf star AD Leonis, which corresponds to the star used in the present study) and of planetary mass in the Earth to super-Earth range, whereas Grenfell et al. (2014) study the effect of varying stellar UV radiation and surface biomass emissions.

One has to be sure to rule out cases where inorganic chemistry can mimic the presence of life ("false positives"). Potential abiotic ozone production on Venus- and Mars-like planets has been discussed by Schindler \& Kasting (2000, and references therein). While this is based on photolysis of e.g., $\mathrm{CO}_{2}$ and $\mathrm{H}_{2} \mathrm{O}$ and is thus limited in extent, a sustainable production of abiotic $\mathrm{O}_{3}$ which could build up to a detectable level has been suggested by Domagal-Goldman \& Meadows (2010) for a planet within the habitable zone of AD Leonis with a specific atmospheric composition. Indeed, other studies confirm that abiotic buildup of ozone is possible (e.g., Hu et al. 2012; Tian et al. 2014); however, detectable levels are unlikely if liquid water is abundant, as e.g. rainout of oxidized species would keep atmospheric $\mathrm{O}_{2}$ and $\mathrm{O}_{3}$ low (Segura et al. 2007), unless the $\mathrm{CO}_{2}$ concentration is high and both $\mathrm{H}_{2}$ and $\mathrm{CH}_{4}$ emissions are low (Hu et al. 2012). False-positive detection of molecules such as $\mathrm{CH}_{4}$ and $\mathrm{O}_{3}$ is discussed by von Paris et al. (2011). Seager et al. (2013) present a biosignature gas classification. Since abiotic processes cannot be ruled out for individual molecules (e.g. for $\mathrm{O}_{3}$ ), searches for biosignature molecules should search for multiple biosignature species simultaneously. It has been suggested that the simultaneous presence of $\mathrm{O}_{2}$ and $\mathrm{CH}_{4}$ can be used as an indication for life (Sagan et al. 1993, and references therein). Similarly, Selsis et al. (2002) suggest a so-called "triple signature", where the combined detection of $\mathrm{O}_{3}, \mathrm{CO}_{2}$ and $\mathrm{H}_{2} \mathrm{O}$ would indicate biological activity. Domagal-Goldman \& Meadows (2010) suggest to simultaneously search for the signature of $\mathrm{O}_{2}, \mathrm{CH}_{4}$, and $\mathrm{C}_{2} \mathrm{H}_{6}$. Of course, care has to be taken to avoid combinations of biosignature molecules which can be generated abiotically together (see e.g. Tian et al. 2014). The detectability of biosignature molecules is discussed, e.g. by von Paris et al. (2011) and Hedelt et al. (2013). In particular, the simulation of the instrumental response to simulated spectra for currently planned or proposed exoplanet characterization missions has shown that the amount of information the retrieval process can provide on the atmospheric composition may not be sufficient (von Paris et al. 2013).

Similar to "false positives", which can lead to erroneous interpretation of observational data, one also has to deal with the problem of "false negatives" for life-bearing planets. The absence of ozone does not necessarily mean that life is absent. Oxygen or ozone may be quickly consumed by chemical reactions, preventing it from reaching detectable levels (Schindler \& Kasting 2000; Selsis et al. 2002). Also, non-detection can result from masking by a wide $\mathrm{CO}_{2}$ absorption (Selsis et al. 2002; von Paris et al. 2011). Here, we look into an abiotic process (namely GCRs) which can destroy the signature of biosignature molecules. Similarly to potential false-positives, these effects have to be taken into account in order to correctly interpret observational data.

As has been shown in Sect. 4.1 (Fig. 4), the ozone column can be modified by up to $13 \%$ by the action of GCRs in the case of weak magnetic fields. We find similar values for other biosignature molecules. In the following, we explore the question: Could this modify the observed spectrum of a planet, either in emission or in transmission?

Figure 5 explores the influence of GCRs on the molecular signature in the planetary spectrum for wavelengths between $2 \leq$ $\lambda \leq 20 \mu \mathrm{m}$ using the SQuIRRL code (cf. Sect. 3.1.4). Figure 5a shows the planetary emission spectrum, whereas Fig. 5b shows the relative transmission coefficient (see Rauer et al. 2011, for details on the spectral methods). In both figures, the black line corresponds to the reference spectrum, i.e. the case of a planet orbiting in the habitable zone of an M dwarf star with no GCRs (zero cosmic-ray case), whereas the red line corresponds to the $\mathrm{M}$ dwarf scenario with GCR and $\mathcal{M}=0.0 \mathcal{M}_{\oplus}$ (i.e. maximum galactic cosmic-ray case). Note that the red line mostly lies over the black line. Both figures indicate that spectral observations would show no detectable difference between the cases with and without GCRs. The same is true for observations at higher spectral resolution (e.g. for a total number of spectral bins $R=10000$, not shown).

We thus reach the conclusion that the influence of GCRs on atmospheric biosignature molecules for Earth-like planets in the habitable zone of M-dwarf stars is too weak to be detectable in the planetary spectra. The case of solar energetic particles is discussed in Tabataba-Vakili et al. (2016).

\subsection{Surface UV flux}

Besides changes in the planetary spectrum, a direct consequence of the loss of stratospheric ozone is an increase in the surface UV radiation, especially in the UV-B range. We would like to know whether this change is important enough to potentially 


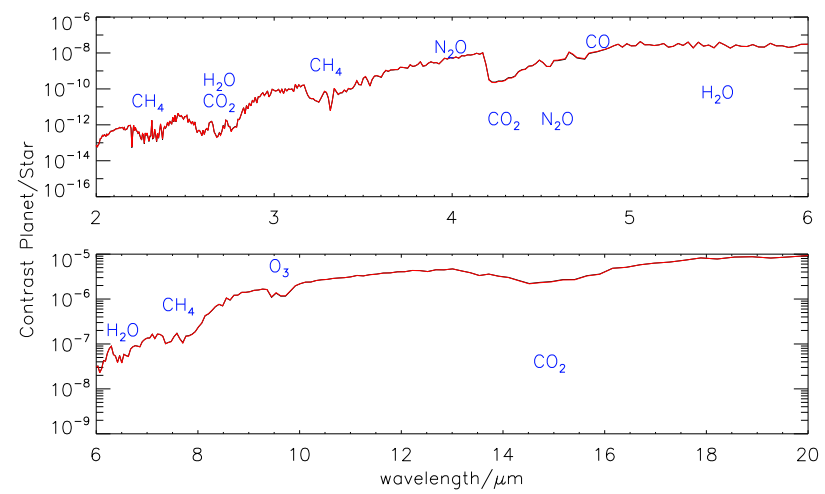

(a) Planetary emission contrast spectrum.

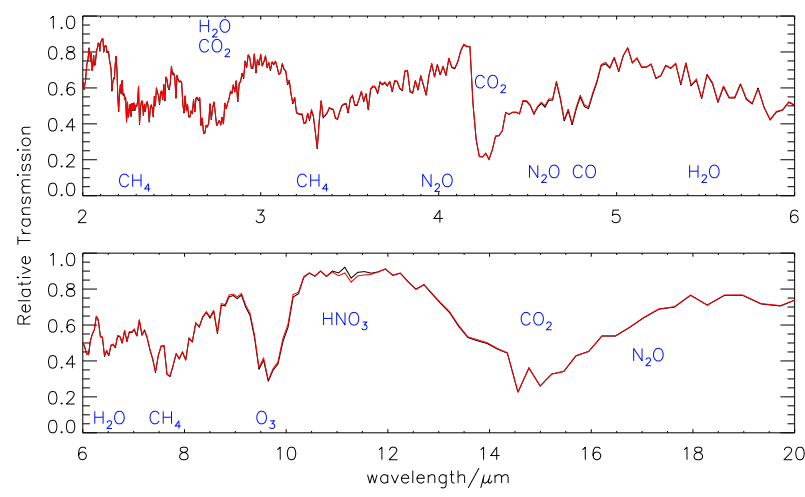

(b) Relative transmission spectrum.

Fig. 5. Emission contrast spectrum (left) and relative transmission spectrum (right) with and without GCRs for an Earth-like planet orbiting in the habitable zone of the M-dwarf star AD Leonis. Black line (mostly hidden under the red line): no GCRs. Red line: maximum GCRs (i.e. no planetary magnetospheric protection). The spectra were calculated for $R=1000$ (total number of spectral bins).

have an impact on life (since most surface life relies on a protective $\mathrm{O}_{3}$-layer).

The atmosphere-penetrating UV radiation is frequently divided into three different ranges:

- UV-A, (3150 to $4000 \AA$ according to the definition of the "Commission Internationale de l'Eclairage", CIE), which has the smallest biological significance;

- UV-B (2800 to $3150 \AA$ according to the CIE definition), which is biologically damaging;

- and UV-C (1754 to $2800 \AA$ in this study), which is strongly damaging, but of which very little reaches the ground for an Earth-like atmosphere.

The biological radiation damage created by UV-A radiation is 5 orders of magnitude weaker than the damage caused by UV-C radiation (Horneck 1995; Cockell 1999; Cuntz et al. 2010). Even so, UV-A still produces significant mutagenic and carcinogenic effects (e.g., de Gruijl 2000; Scalo et al. 2007). Due to very efficient atmospheric shielding, the surface flux of UV-C is usually many orders of magnitude smaller than that of UV-A or UV-B, even during stellar flares (Segura et al. 2010). For this reason, most work (e.g. Grenfell et al. 2012) concentrates on UV-B radiation (280-315 nm). In this work, however, we proceed slightly differently. Similarly to Segura et al. (2010), we study the full UV spectrum from 1754-3150 $\AA$, and present integrated results for the two relevant bands UV-A and UV-B. The surface UV-C fluxes turn out to be negligible due to atmospheric shielding.

One should note that the ways in which UV radiation can be harmful for living cells are complex and varied (e.g., de Gruijl 2000; Scalo et al. 2007, and references therein). In addition, some species have found ways to protect themselves against harmful radiation, either through repair mechanisms (Scalo et al. 2007), protective layers, or through strategies which allow to avoid strong radiation altogether (e.g. Sect. 7 of Heath et al. 1999; Scalo et al. 2007). The influence of a highly fluctuating environment on life is discussed by Scalo et al. (2007, and references therein).

The UV flux of different M-dwarf stars can differ considerably, and is highly variable in time (France et al. 2013). Grenfell et al. (2014) investigate the effect of different stellar UV fluxes on atmospheric biosignatures. When analyzing the flux of the UV radiation of an M-dwarf star to the planetary surface, we have to distinguish between a number of different cases: the quiescent stellar UV flux of a non-active star, the UV flux of a chromospherically active star (such as AD Leo or GJ 643C), and the UV flux during a stellar UV flare. For the latter case, we differentiate between "long" and "short" flares, depending on the flare duration relative to the atmospheric response timescale (see below). Thus, the relevant cases are:

E) We compare to the case of Earth in the habitable zone of the Sun (i.e. at a distance of $1 \mathrm{AU})$.

Q) The quiescent emission of (model) non-active stars is characterized by UV fluxes many orders of magnitude smaller than for the Sun over the whole UV range (Segura et al. 2005). Recent studies indicate that this case may be less representative than previously thought (France et al. 2013). No noticeable effect is expected and thus this case is not further discussed in this work.

CA) Chromospherically active stars have additional flux in the range $100-300 \mathrm{~nm}$, generated by chromospheric and coronal activity. Although their absolute UV flux is inferior to that of the Sun (e.g. Buccino et al. 2007, Fig. 1), their normalized UV flux (i.e. at a distance where the surface temperature or total flux equals that on present Earth) however may exceed the solar value (e.g. by a factor up to 10 in UV-C, Segura et al. 2005). Recent studies indicate that AD Leo may be more active than previously thought (France et al. 2013). For a planet in the habitable zone of a chromospherically active M-dwarf star, we use the top of atmosphere (TOA) UV spectrum of AD Leonis, as described in Sect. 3.1.

LF) During a stellar flare, the UV flux typically increases by one order of magnitude (France et al. 2013), and by at least two orders of magnitude in more extreme cases (Scalo et al. 2007; Segura et al. 2010, who looked at different stages of the 1985 flare of AD Leo), with a timescale of $10^{2}-10^{3} \mathrm{~s}$. For a planet in the habitable zone of a flaring M-dwarf star, we use the TOA UV spectrum of Segura et al. (2010, their Fig. 3, bold blue line, scaled for distance), i.e. the maximum flux at the flare peak. The TOA flux is approximately solar for UV-A and 2.5 times solar for UV-B (see Table 1). For UV-C, the TOA flux is $\sim 10$ times solar. The timescale of the flare has to be compared to the timescale over which the atmosphere responds. As we are using a stationary model, we can only probe the extreme cases of very long (quasicontinuous) stellar UV flares, where the time between flares is so short that the atmosphere is constantly under flaring conditions, and of very short (isolated) flares, where the atmosphere does not have the time to react to the flare. In the 
J.-M. Grießmeier et al.: Galactic cosmic rays on extrasolar Earth-like planets. II.

Table 1. UV-A and UV-B flux at top of atmosphere $\left(I_{\mathrm{TOA}}\right)$ and the surface $\left(I_{\text {surface }}\right)$ for different cases, wavelength-range averaged flux ratio $R=I_{\text {surface }} / I_{\text {TOA }}$ (i.e. atmospheric transmission coefficient), and biologically weighted surface UV flux $W$ (in weighted W/ $/ \mathrm{m}^{2}$ ).

\begin{tabular}{lc|ccc|ccc|c}
\hline \hline Column 1 & Column 2 & 3 & 4 & 5 & 6 & 7 & 8 & 9 \\
\hline Case & $\begin{array}{c}\mathcal{M} \\
{\left[\mathcal{M}_{\oplus}\right]}\end{array}$ & $\begin{array}{c}I_{\text {TOA }}^{\mathrm{UV}-\mathrm{A}} \\
{\left[\mathrm{W} / \mathrm{m}^{2}\right]}\end{array}$ & $\begin{array}{c}I_{\text {surface }}^{\mathrm{UV}-\mathrm{A}} \\
{\left[\mathrm{W} / \mathrm{m}^{2}\right]}\end{array}$ & $R^{\mathrm{UV}-\mathrm{A}}$ & $\begin{array}{c}I_{\text {TOA }}^{\mathrm{UV}-\mathrm{B}} \\
{\left[\mathrm{W} / \mathrm{m}^{2}\right]}\end{array}$ & $\begin{array}{c}I_{\text {surface }}^{\mathrm{UV}-\mathrm{W}} \\
{\left[\mathrm{W} / \mathrm{m}^{2}\right]}\end{array}$ & $R^{\mathrm{UV}-\mathrm{B}}$ & $\begin{array}{c}W \\
{\left[\mathrm{~W} / \mathrm{m}^{2}\right]}\end{array}$ \\
\hline E & 1.0 & 127 & 90.41 & 0.71 & 18.29 & 2.264 & 0.12 & 0.126 \\
E & no GCR & 127 & 90.41 & 0.71 & 18.29 & 2.256 & 0.12 & 0.125 \\
CA & 0.0 & 2.01 & 1.479 & 0.74 & 0.202 & 0.0225 & 0.11 & 0.0021 \\
CA & 0.1 & 2.01 & 1.479 & 0.74 & 0.202 & 0.0221 & 0.11 & 0.0020 \\
CA & 1.0 & 2.01 & 1.477 & 0.74 & 0.201 & 0.0204 & 0.10 & 0.0016 \\
CA & 10.0 & 2.01 & 1.477 & 0.74 & 0.200 & 0.0198 & 0.10 & 0.0015 \\
CA & no GCR & 2.01 & 1.477 & 0.74 & 0.200 & 0.0197 & 0.10 & 0.0015 \\
LF & 0.0 & 112 & 75.75 & 0.68 & 47 & 3.053 & 0.065 & 0.145 \\
LF & 0.1 & 112 & 75.75 & 0.68 & 47 & 3.054 & 0.065 & 0.145 \\
LF & 1.0 & 112 & 75.79 & 0.68 & 47 & 3.084 & 0.066 & 0.148 \\
LF & 10.0 & 112 & 75.80 & 0.68 & 47 & 3.099 & 0.066 & 0.149 \\
LF & no GCR & 112 & 75.81 & 0.68 & 47 & 3.100 & 0.066 & 0.150 \\
SF & 0.0 & 112 & 76.50 & 0.68 & 47 & 5.10 & 0.11 & 0.55 \\
SF & 0.1 & 112 & 76.47 & 0.68 & 47 & 5.00 & 0.11 & 0.52 \\
SF & 1.0 & 112 & 76.37 & 0.68 & 47 & 4.65 & 0.10 & 0.42 \\
SF & 10.0 & 112 & 76.33 & 0.68 & 47 & 4.52 & 0.10 & 0.39 \\
SF & no GCR & 112 & 76.33 & 0.68 & 47 & 4.51 & 0.10 & 0.39 \\
\hline
\end{tabular}

Notes. Cases: $\mathrm{E}=$ Earth, $\mathrm{CA}=$ chromospherically active star, $\mathrm{LF}=$ long flare, $\mathrm{SF}=$ short flare (see text for details).

case of a long flare, the flare timescale is longer than the typical reaction time of the planetary atmosphere and the atmosphere adjusts to the modified conditions. In this case, we calculate the surface UV flux from the modified TOA flux using the model of Sect. 3.1. This case also applies when the planet is subject to a quasi-continuous succession of UV flares, which might well be the case for planets around active M-dwarf stars (Khodachenko et al. 2007; Grenfell et al. 2012).

$\mathrm{SF}$ ) If, on the other hand, the timescale of the UV flare (e.g. its duration) is short compared to the atmospheric reaction time, the atmosphere has not yet adjusted to the increased UV flux. In this short flare case, the atmosphere, and thus its transmission ratio $R(\lambda)=I_{\text {surface }}(\lambda) / I_{\mathrm{TOA}}(\lambda)$ are identical to the preflare conditions, i.e. the case CA described above. Hereby, $I_{\text {surface }}(\lambda)$ and $I_{\mathrm{TOA}}(\lambda)$ denote the flux at the planetary surface and at the top of the atmosphere, respectively. The top-ofatmosphere UV-flux, however, is identical to the flaring case, LF. We thus use the transfer function $R(\lambda)$ obtained in the case CA, and multiply it with the TOA UV flux of the case LF (Segura et al. 2010, their Fig. 3, bold blue line scaled for distance) to obtain the surface UV flux. On Earth and in our exoplanetary calculations, most atmospheric ozone is located below $50 \mathrm{~km}$ where reaction timescales are long (e.g. Allen et al. 1984), so that this scenario is appropriate for an isolated flare (i.e. the flare timescales are much shorter than the atmospheric reaction timescales).

SCR) Stellar UV flares are expected to be frequently accompanied by stellar cosmic-ray (SCR) particles, which are not included in the above cases LF and SF. The influence of such SCRs (as opposed to the GCRs discussed in the present work) are only briefly discussed below. A more detailed analysis is presented separately (Tabataba-Vakili et al. 2016).

In addition to the UV flux emitted by the planetary host star, another source can contribute to atmospheric and surface UV. Smith et al. (2004) have suggested that stellar X-rays may be reprocessed in the atmosphere, generating an additional contribution of UV photons. They found that up to $10 \%$ of the X-ray energy may be redistributed into the UV range by aurora-like emission in the absence of UV-blocking agents (i.e. when the UV transport is defined by Rayleigh scattering alone). In the case of the Earth, UV redistribution may transfer a fraction of $2 \times 10^{-3}$ of the incident energy to the planetary surface in the 200-320 nm range. Segura et al. (2010) estimated the X-ray energy for a strong flare on the M-dwarf star AD Leo flare to be $9 \mathrm{~W} / \mathrm{m}^{2}$, so the energy redistributed as UV radiation at the planetary surface should be $<0.018 \mathrm{~W} / \mathrm{m}^{2}$. This is negligible compared to the UV flux of the flare itself (cf. Table 1).

For the above cases E), CA) and LF), we are interested in the transmission of UV radiation through the atmosphere. For each case, we investigate the full range from $\mathcal{M}=0$ (i.e. no magnetospheric shielding, where the atmospheric ozone is most strongly depleted by galactic cosmic rays) up to $10 \mathcal{M}_{\oplus}$ (i.e. strong magnetospheric shielding), plus the case without GCRs (which, for our purposes, corresponds to a planet with an infinite magnetic moment, and thus a planet with maximum stratospheric ozone shield).

We proceed as follows:

- We take the wavelength-resolved stellar UV flux and calculate the flux incident at the top of atmosphere (TOA) of the M-dwarf star planet corresponding to the planetary orbital distance. From this we calculate the wavelength-integrated TOA UV fluxes $I_{\mathrm{TOA}}^{\mathrm{UV}-\mathrm{A}}$ and $I_{\mathrm{TOA}}^{\mathrm{UV}-\mathrm{B}}$ (Cols. 3 and 6 of Table 1).

- With this UV flux, the numerical model described in Sect. 3.1, and using the magnetic-field dependent TOA fluxes of GCR particles from Paper I, we calculate the wavelength-resolved flux of UV at the planetary surface. From this we calculate the wavelength-integrated surface UV fluxes $I_{\text {surface }}^{\mathrm{UV}-\mathrm{A}}$ and $I_{\text {surface }}^{\mathrm{UV}}($ Cols. 4 and 7 of Table 1). The effects that are considered here are absorption (by $\mathrm{O}_{3}$ and other species) and Rayleigh scattering by atmospheric molecules.

- We calculate the ratio of UV penetrating through the planetary atmosphere, averaged over the corresponding $\mathrm{UV}$ band, e.g. $R^{\mathrm{UV}-\mathrm{A}}=I_{\text {surface }}^{\mathrm{UV}} / I_{\mathrm{TOA}}^{\mathrm{UV}-\mathrm{A}}$, and similarly for $R^{\mathrm{UV}-\mathrm{B}}$ (Cols. 5 and 8 of Table 1 ). $R$ thus characterizes the average UV shielding by the atmosphere in a particular wavelength band. Note that the value of $R$ depends on both the TOA GCR 


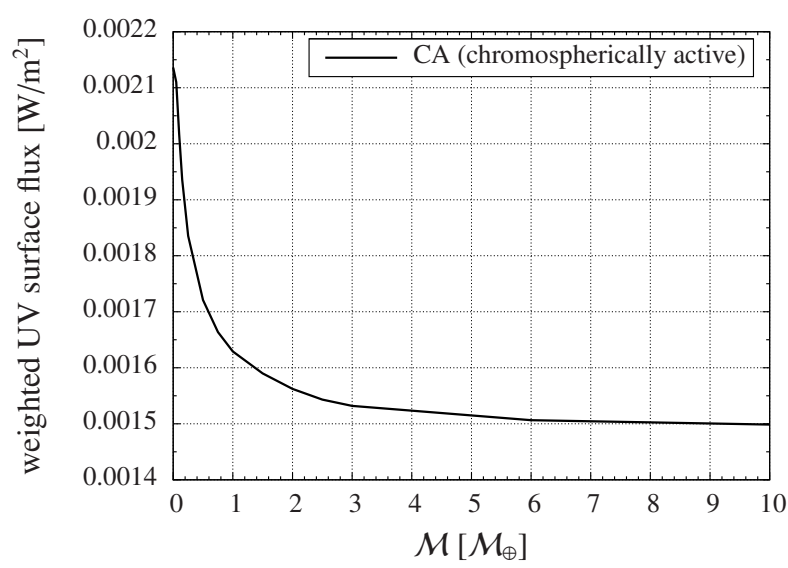

Fig. 6. Biologically weighted surface UV flux $W$ as a function of magnetospheric shielding for a chromospherically active star (case CA).

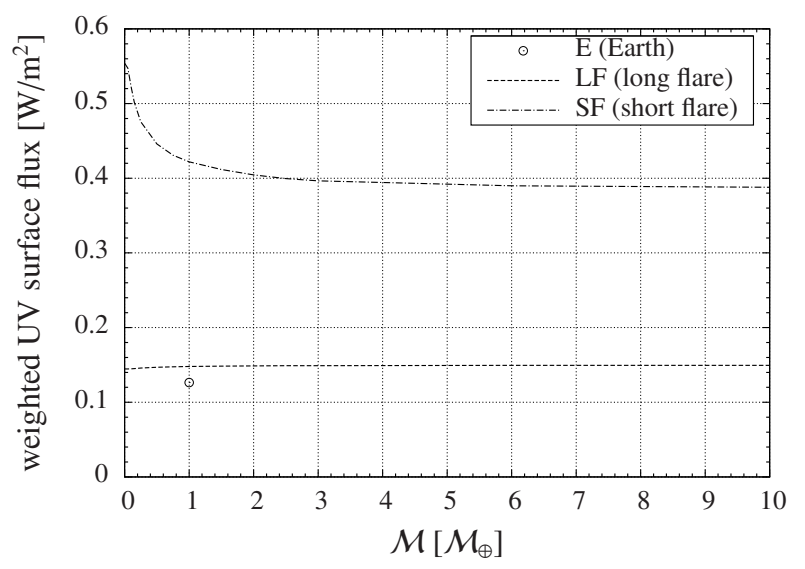

Fig. 7. Biologically weighted surface UV flux $W$ as a function of magnetospheric shielding. Dashed line: long flare (case LF). Dash-dotted line: short flare (case SF). Circle: Earth (case E).

flux and the TOA UV flux (the atmosphere behaves as a nonlinear system).

- We multiply the wavelength-resolved UV surface spectra with the DNA action spectrum of Cuntz et al. (2010, Fig. 1), which is based on previously published data (Horneck 1995; Cockell 1999), to calculate the effective biological UV flux $W$ at the planetary surface (Col. 9 of Table 1, and Figs. 6 and 7). In this, the DNA action spectrum is normalized to 1 at a wavelength of $300 \mathrm{~nm}$ (i.e. at $300 \mathrm{~nm}$, a flux of $1 \mathrm{~W} / \mathrm{m}^{2}$ contributes $1 \mathrm{~W} / \mathrm{m}^{2}$ to $W$ ). We also compare the relative contribution of UV-A, UV-B and UV-C to the effective biological UV flux $W$.

Our main results are described in the following (see also Table 1).

Case E (Earth): in the case of the Earth, GCRs leave both the UV transmission coefficients and the UV surface fluxes virtually unchanged. As a consequence, the biologically weighted UV surface flux $W$ is barely affected by the presence of GCRs (Table 1, Col. 9).

The surface UV-B results, i.e. the surface flux (Table 1, Col. 7) and the transmission coefficient (Table 1, Col. 8) show a good accordance with Grenfell et al. (2012, Table 2, line 1 ), where $R^{\mathrm{UV}-\mathrm{B}}=0.13$ for the case with GCRs, and with Grenfell et al. (2013), where $R^{\mathrm{UV}-\mathrm{B}}=0.16$ for the case without
GCRs. These values are also compatible with Earth observations (Grenfell et al. 2012, Table 2, line 4).

For the biologically weighted flux, we find $W=$ $0.126 \mathrm{~W} / \mathrm{m}^{2}$, which is dominated by the contribution of UV-B (94\%), with a small contribution from UV-A (6\%). The influence of UV-C on $W$ is negligible. Cockell (1999) obtain a lower value for $W$, which is possibly due to their stronger ozone layer.

Case CA (chromospherically active star): in the case of a planet in the habitable zone of a chromospherically active Mdwarf star, the transmission ratio for UV-A is $R^{\mathrm{UV}-\mathrm{A}}=0.74$ (Table 1, Col. 5), independent of magnetic shielding, and similar to the case of the Earth.

As shown in Table 1, the cosmic-ray induced weakening of the ozone layer described in Sect. 4.1 has little influence on the atmospheric UV-B transmission ratio $R^{\mathrm{UV}-\mathrm{B}}$ (Col. 8), which increases from 0.10 to 0.11 with decreasing magnetic shielding (i.e. increasing GCR effect). Between strong and zero magnetic shielding, the UV-B surface flux increases by $14 \%$ (for a decrease of the ozone column by $13 \%$, see Sect. 4.1). This is consistent with the near-linear relationship between atmospheric ozone column and surface UV-B flux observed on Earth (e.g. Kerr \& McElroy 1993). Due to the low intensity of UV-B emitted by the M-dwarf star, the resulting UV-B surface flux is very small (two order of magnitude less than on present-day Earth, see Col. 7).

The surface UV-C flux (not shown) is negligibly small, even when the high biological response factor to UV-C is taken into account.

Figure 6 shows how the biologically weighted UV surface flux $W$ changes as a function of magnetic shielding; between minimum and maximum magnetic shielding, $W$ changes by $\sim 40 \%$. It is dominated by the contribution of UV-B ( $\geq 95 \%)$, with a small contribution from UV-A (3-5\%). The influence of UV-C on $W$ is negligible. A detailed analysis shows that for our parameters the UV-B flux at $300 \mathrm{~nm}$ has the strongest contribution to $W . W$ is considerably lower than on Earth (case E; see Col. 9 of Table 1). Our results are in good agreement with Grenfell et al. (2013), who find $R^{\mathrm{UV}-\mathrm{B}}=0.11$ for AD Leo (case without cosmic rays).

Case LF (long stellar UV flare): as shown in Table 1, the results are different in the case of an exoplanet either exposed to a long UV flare or to a quasi-continuous succession of UV flares (case LF). The UV-A transmission ratio $R^{\mathrm{UV}-\mathrm{A}}$ is similar to that of the case CA, but the higher TOA flux leads to an increased surface UV-A intensity.

For UV-B, the transmission ratio is reduced compared to the case CA. However, this is compensated by the increased TOA flux, so that the surface flux is higher than for the chromospherically active case (about two orders of magnitude) and exceeds the level of case $\mathrm{E}$.

Again, the surface UV-C flux (not shown) is negligibly small, even when the high biological response factor to UV-C is taken into account.

Compared to the case CA, the biologically weighted UV surface flux $W$ (which is mostly determined by shortwave UV-B) is increased by up to two orders of magnitude in the case LF. It is comparable to the value for Earth (case E, circle in Fig. 7. See also Col. 9 of Table 1). $W$ is dominated by the contribution of UV-B $(\geq 92 \%)$, with a small contribution from UV-A $(7-8 \%)$. The influence of UV-C on $W$ is negligible. 

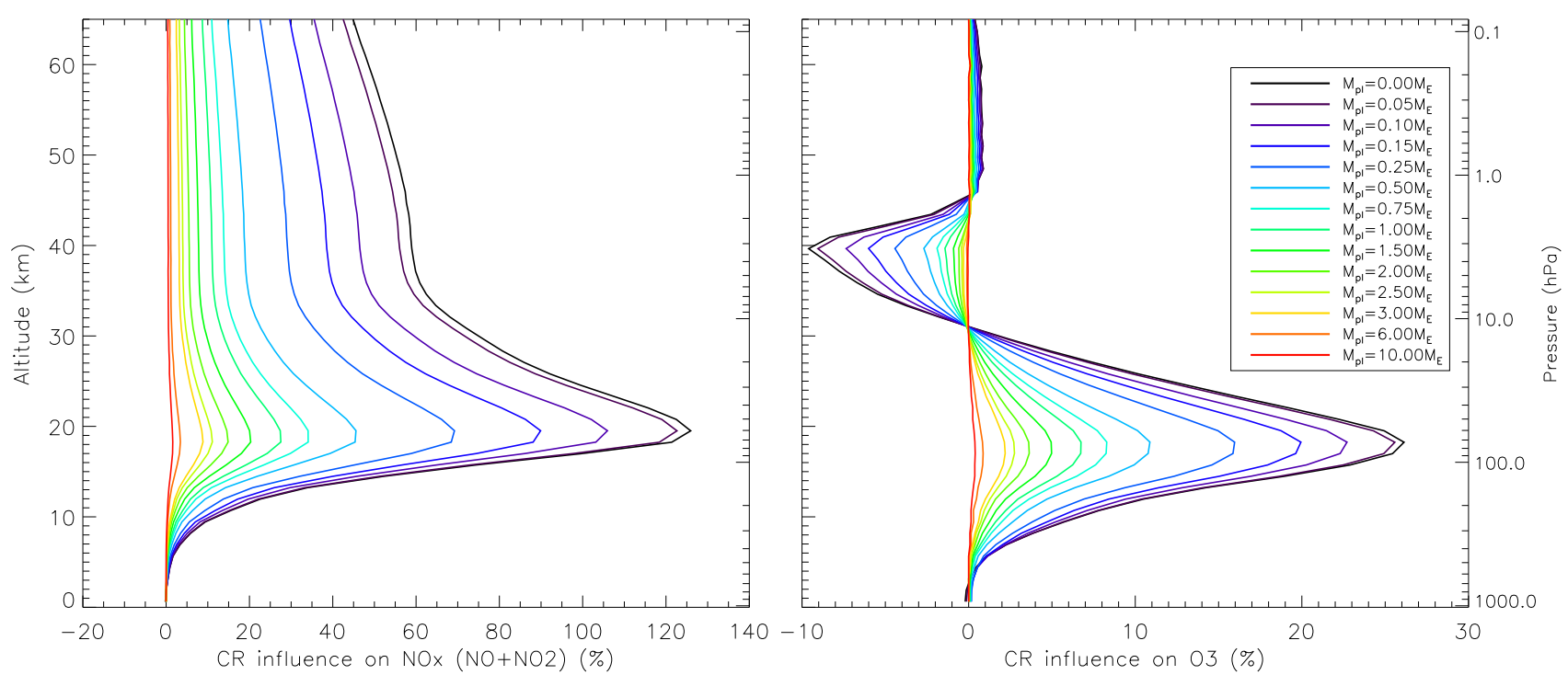

Fig. 8. As Fig. 2 but for the case of a long UV flare (LF).

Another effect of long flares can be seen in Table 1 and Fig. 7: when the magnetic moment increases, i.e. when the planet is better shielded against GCRs, the surface UV flux shows a slight increase! This is a consequence of the enhanced stellar UV flux, which leads to extra ozone production in the altitude region 5-30 km, with a peak at $18 \mathrm{~km}$ (Fig. 8). This suggests an increasing smog mechanism at low altitudes, whereas above $30 \mathrm{~km} \mathrm{O}_{3}$ is still destroyed by catalytic $\mathrm{NO}_{x}$. Figure 8 shows that with increasing GCR flux (decreasing magnetic shielding), the smog mechanism in the lower atmosphere dominates over the catalytic destruction in the upper atmosphere, so that the column integrated ozone content increases, and the surface UV flux decreases (Fig. 7).

Case SF (short stellar UV flare): for a short stellar flare (with a timescale of $10^{2}-10^{3} \mathrm{~s}$ ), we do not have the inversed response (i.e. increase of surface UV with increasing magnetic moment) of case LF, but instead (by construction) a behavior identical to the case CA, with higher absolute flux values (cf. Table 1). Also, by construction, the averaged UV transmission rates are similar to the case CA and the atmospheric profile is identical to that of the case CA (Fig. 2).

As a result of the higher input flux, the surface flux of UV-A and UV-B is at least 50 times higher than in the case CA, see Table 1.

In the case SF, the biologically weighted UV surface flux $W$ is higher than in the case CA by a factor 200-300. Again, $W$ is dominated by the contribution of UV-B ( $\geq 97 \%)$, with a small contribution from UV-A (2-3\%). The influence of UV-C on $W$ is negligible.

The modulation by the magnetic field is comparable to the case CA (compare Figs. 6 and 7): between $\mathcal{M}=0$ and $1.0 \mathcal{M}_{\oplus}$, $W$ decreases by $30 \%$. For higher magnetic fields, $W$ continues to decrease, by up to $10 \%$ (Fig. 7). As indicated in Table 1 (Col. 9) and Fig. 7, $W$ is a factor of 3-4 higher than on Earth (case E) for the duration of the short flare.

Case SCR (stellar cosmic rays): UV flares are frequently accompanied by stellar cosmic-ray particles (Segura et al. 2010), which would amplify the ozone destruction. In that case, the strong removal of stratospheric ozone (Grenfell et al. 2012) can reduce the UV shielding to $\sim 50 \%$, and lead to considerably higher UV surface fluxes, surpassing those on the terrestrial surface by an order of magnitude. Using the current model, this case is re-evaluated in a separate article (Tabataba-Vakili et al. 2016).

Discussion: the comparison shows that a short flare is potentially more harmful than a long flare, in which the atmosphere has time to adjust to the high UV flux and absorption is increased at mid-altitudes. The effect of GCRs on planetary UV radiation is weaker than the modification caused by a change in the stellar spectrum (e.g. case E to CA). Looking at the different wavelength ranges, one notices that:

- GCRs leave the UV-A transmission coefficient and flux virtually unchanged.

- For UV-B, GCRs modify the transmission rate and surface flux by less than $20 \%$, and the relative change is proportional to the change in ozone column, as expected.

- The surface flux of UV-C remains negligibly small in all cases.

- GCRs may change the biologically weighted UV surface flux $W$ by up to $40 \%$. In all cases, $W$ is dominated by the contribution of UV-B $(\geq 90 \%)$, with a minor contribution from UV-A (2-8\%). UV-C does not contribute significantly to $W$.

- The GCR-induced variation of $W(40 \%)$ is much less than the difference due to a change in stellar emission during a flare. For example, during a short stellar flare (case SF), one finds values 3-4 times higher than on Earth (case E), or 200-300 times the quiescent level (case CA).

- The GCR-induced UV radiation is $W \leq 0.55 \mathrm{~W} / \mathrm{m}^{2}$ (Table 1 , Col. 9). This value has to be compared to the tolerance of biological systems. In particular, Deinoccocus radiodurans is able to withstand high levels of UV radiation. Gascón et al. (1995) estimate the $\mathrm{D}_{90}$ dose (i.e. the dose for inactivation of $90 \%$ of the bacterial population) of Deinoccocus radiodurans to be $\sim 553 \mathrm{~J} / \mathrm{m}^{2}$. For their measurements, they used a UV lamp with a flux density of $1.7 \mathrm{~W} / \mathrm{m}^{2}$ at $256 \mathrm{~nm}$; the $\mathrm{D}_{90}$ time their case was thus of the order of $5 \mathrm{~min}$. The flux density of their lamp corresponds to an equivalent DNA effective irradiance of $43 \mathrm{~W} / \mathrm{m}^{2}$ (Cockell 1999), i.e. almost 
two order of magnitude stronger than the maximum UV flux caused by GCRs, cf. Table 1 . The $\mathrm{D}_{90}$ time for Deinoccocus radiodurans on the surface of a magnetically unshielded exoplanet exposed to GCRs plus stellar UV flares would thus be of the order of $7 \mathrm{~h}$. Also, bacteria are usually not fully exposed. Cockell (1999) estimates that life on Earth may have arisen during times when the biologically weighted UV flux was $>96 \mathrm{~W} / \mathrm{m}^{2}$, which is higher by a factor 170 than the maximum value for GCR-induced UV ( $W \leq 0.55 \mathrm{~W} / \mathrm{m}^{2}$, cf. Table 1). We thus conclude that GCR-induced UV radiation can be considered as non-critical.

We conclude that GCR-induced UV radiation is non-critical, but we note that the UV environment on M-dwarf star planets is much more variable than on Earth.

\subsection{Surface biological dose rate}

In this section, we analyze which fraction of cosmic-ray particles can reach the planetary surface, and evaluate the associated biological dose rate.

Like on Earth, the surface of an exoplanet can be shielded against galactic cosmic rays by two barriers. The first barrier is the planetary magnetosphere, which deflects particles provided their energy is low enough (Paper I). However, this does not mean that all particles that penetrate through the magnetosphere reach the surface. The atmosphere acts as a second barrier, and prevents low-energy particles and their products from reaching the surface (O'Brien et al. 1996). At Earth, the minimum energy a proton must have to initiate a nuclear interaction sequence detectable at the surface is approximately $450 \mathrm{MeV}$ (Shea \& Smart 2000). Higher energy protons generate an atmospheric nuclear cascade or cosmic-ray shower, with high energy secondary particles such as neutrons, electrons, pions and muons reaching the planetary surface. The low-energy components of the cascade are absorbed in the atmosphere, leading to an altitude with maximum particle flux, the Pfotzer maximum. In the case of the Earth, the Pfotzer maximum is located at an altitude of $15-26 \mathrm{~km}$, depending on latitude and solar activity level (Bazilevskaya et al. 2008). Below the Pfotzer maximum, the particle flux decreases toward the surface. Depending on the altitude of the Pfotzer maximum, the surface radiation dose can either be lower or higher than at the top of the atmosphere. For a planet with an Earth-like atmosphere, the absorption effect dominates, and the atmosphere has to be regarded as a second barrier which partially protects the surface against the cosmic-ray flux.

If parts of the cosmic-ray shower reach the planetary surface, one could expect that biological systems there can be strongly influenced and even damaged by this secondary radiation. This expectation is backed up by experimental evidence, which shows that during Ground Level Enhancements (extreme events where large numbers of secondary cosmic rays reach the Earth's surface) DNA lesions on the cellular level increase considerably (Belisheva et al. 2005; Grießmeier et al. 2005; Belisheva et al. 2006, 2012; Dartnell 2011, and references therein). In the case of Earth, muons contribute $75 \%$ of the equivalent dose rate at the surface (O'Brien et al. 1996).

In Paper I, we have shown that weakly magnetized superEarths orbiting M-dwarf stars can be exposed to much higher cosmic-ray fluxes at the top of the atmosphere when compared to the case of the Earth. The question naturally arises: how does this high flux at the top of the atmosphere translate into a radiation dose at the planetary surface?

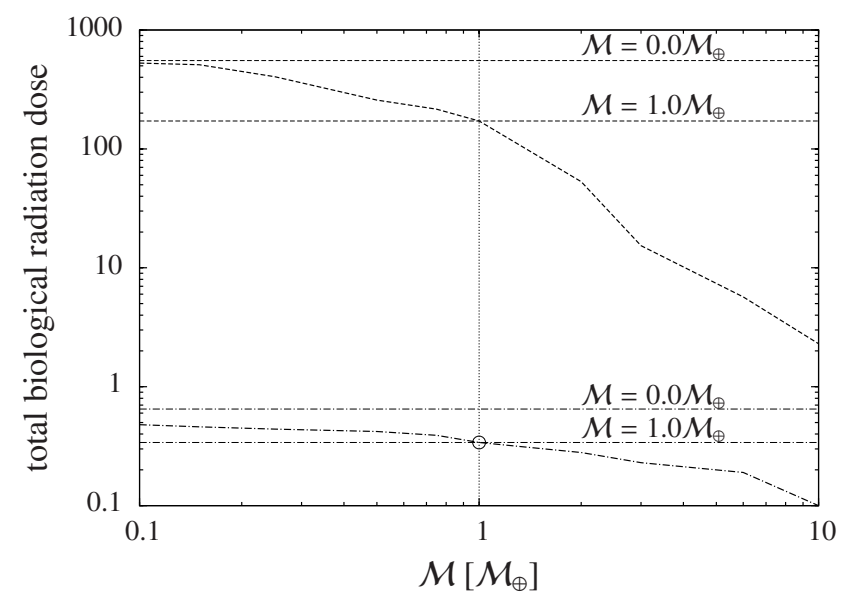

Fig. 9. Total biological dose rate (i.e. the sum of radiation dose rates by muons, electrons, and neutrons, in $\mathrm{mSv} / \mathrm{yr}$ ) as a function of magnetospheric shielding. Bold dash-dotted line: planet with an atmospheric depth of $1036 \mathrm{~g} / \mathrm{cm}^{2}$ (i.e. an Earth-like atmosphere with a surface pressure of $1033 \mathrm{hPa}$ ). Bold dashed line: planet with an atmospheric depth of $100 \mathrm{~g} / \mathrm{cm}^{2}$ (i.e. a surface pressure of $97.8 \mathrm{hPa}$ ). Vertical line (shown as a guide for the eye): $\mathcal{M}=1.0 \mathcal{M}_{\oplus}$. Horizontal lines (shown for comparison): Total biological dose rates for the case $\mathcal{M}=0$ (upper dashed and upper dash-dotted horizontal line) and $\mathcal{M}=1.0 \mathcal{M}_{\oplus}$ (lower dashed and lower dash-dotted line). Circle: Earth.

Table 2. Total biological dose rate $B$ (i.e. the sum of radiation dose rates by muons, electrons, and neutrons, in $\mathrm{mSv} / \mathrm{yr}$ ) in the case of modified magnetospheric shielding for a planet with an atmospheric depth of $1036 \mathrm{~g} / \mathrm{cm}^{2}\left(B^{1036}\right)$ and for a planet with an atmospheric depth of $100 \mathrm{~g} / \mathrm{cm}^{2}\left(B^{100}\right)$.

\begin{tabular}{ccc}
\hline \hline $\mathcal{M}\left[\mathcal{M}_{\oplus}\right]$ & $B^{1036}[\mathrm{mSv} / \mathrm{yr}]$ & $B^{100}[\mathrm{mSv} / \mathrm{yr}]$ \\
\hline 0.0 & 0.65 & 553 \\
0.1 & 0.48 & 527 \\
0.15 & 0.46 & 510 \\
0.25 & 0.44 & 405 \\
0.5 & 0.42 & 257 \\
0.75 & 0.39 & 216 \\
1.0 & 0.34 & 172 \\
2.0 & 0.28 & 53 \\
3.0 & 0.23 & 15 \\
6.0 & 0.19 & 5.7 \\
10.0 & 0.1 & 2.3 \\
\hline
\end{tabular}

The details of this interaction and the resulting radiation dose on the planetary surface depend on the planetary atmospheric pressure and composition. Thus, the best way to address this issue is to simulate numerically the interactions by following the particles from the top of the atmosphere down to the planetary surface. For this, we use the surface particle flux and radiation dose model as described in Sect. 3.2. First results have been presented by Atri et al. (2013); for the current work, the range of planetary magnetic moments has been extended.

Figure 9 and Table 2 show the total biological radiation dose rate as a function of the planetary magnetic field, measured in $\mathrm{mSv} / \mathrm{yr}$. In Fig. 9, the dash-dotted line corresponds to a planet with an atmospheric depth of $1036 \mathrm{~g} / \mathrm{cm}^{2}\left(B^{1036}\right.$, i.e. the biological radiation dose rate for an Earth-like atmosphere with a surface pressure of $1033 \mathrm{hPa}$ ), while the dashed line corresponds to a planet with an atmospheric depth of $100 \mathrm{~g} / \mathrm{cm}^{2}\left(B^{100}\right.$, the biological radiation dose rate for a planet with a surface pressure of $97.8 \mathrm{hPa}$ ). The vertical line denotes Earth's magnetic moment $\left(\mathcal{M}=1.0 \mathcal{M}_{\oplus}\right)$, and the circle indicates Earth-like conditions. 
The horizontal lines are shown to guide the eye. They indicate the total biological dose rates for the cases $\mathcal{M}=0$ (upper dashed and upper dash-dotted horizontal line) and $\mathcal{M}=1.0 \mathcal{M}_{\oplus}$ (lower dashed and lower dash-dotted horizontal line).

In the case of a planet with an Earth-like atmosphere with a surface pressure of $1033 \mathrm{hPa}$ (dash-dotted line), magnetospheric shielding reduces the surface biological dose rate by a factor of approximately 2 between $\mathcal{M}=0$ and $1 \mathcal{M}_{\oplus}$. Obviously, for stronger magnetic fields $\left(\mathcal{M}>1 \mathcal{M}_{\oplus}\right)$, the biological dose rate further decreases (by another factor of 3 for $\mathcal{M}=10.0 \mathcal{M}_{\oplus}$ ). As the magnetic field decreases, the filter efficiency of the magnetosphere decreases, and the number of cosmic-ray protons reaching the top of the planetary atmosphere increases (Paper I). However, the atmosphere remains as a second filter, and removes most of the biologically relevant particles, so that the total biological radiation dose rate of Fig. 9 (i.e. the sum of the radiation dose rates by muons, electrons, and neutrons) increases only slowly with decreasing magnetic moment.

For a planet with a weaker atmosphere having a surface pressure of $97.8 \mathrm{hPa}$ (dashed line), magnetospheric shielding is more important, and reduces the surface biological dose rate by a factor of approximately 3 between $\mathcal{M}=0$ and $1 \mathcal{M}_{\oplus}$, and another factor of 70 between $\mathcal{M}=1$ and $10 \mathcal{M}_{\oplus}$.

As was already noted by Atri et al. (2013), atmospheric shielding dominates over magnetospheric shielding. In Fig. 9 and Table 2, this is indeed obvious: at $\mathcal{M}=0$, the Earth-like atmosphere (bold dash-dotted curve in Fig. 9) reduces the surface biological dose rate by almost three orders of magnitude when compared to the weak atmosphere case (bold dashed curve). For an Earth-like magnetic moment $\left(\mathcal{M}=1 \mathcal{M}_{\oplus}\right)$, the difference is still more than two orders of magnitude. For strongly magnetized planets, the difference is smaller, but atmospheric shielding still remains stronger than the magnetospheric shielding.

The values of Table 2 have to be compared to the terrestrial background radiation of $2.4 \mathrm{mSv} \mathrm{yr}^{-1}$ (Atri et al. 2013). A planet with a sufficiently thick atmosphere (where, for example, an Earth-like $\mathrm{N}_{2}-\mathrm{O}_{2}$-atmosphere with a surface pressure of a $\sim 1$ bar can be considered as "sufficiently thick") is protected against strong biological radiation generated by GCR regardless of its magnetic field. For planets with a thin atmosphere, however, magnetospheric shielding is important as it can prevent an increase of the radiation dose to several hundred times the background level. For close-in rocky planets less massive than Earth, atmospheric escape can play an important role, so that the planetary atmosphere is likely to be less dense than on Earth. For this reason, our result is likely to be important for potential life on the surface of sub-Earth-mass close-in rocky exoplanets.

\section{Conclusion}

Magnetic fields on most super-Earths around M-dwarf stars are likely to be weak and short-lived, or even non-existent. With this in mind, the question of planetary magnetic shielding against galactic cosmic rays becomes important (the case of stellar cosmic rays is analyzed in a separate article, Tabataba-Vakili et al. 2016). We use the systematic study of GCR fluxes presented in Paper I, where we found that the flux of galactic cosmic rays to the planetary atmosphere can be increased by over three orders of magnitude in the absence of a protecting magnetic field.

With this input, we found that these energetic particles can destroy part of the atmospheric ozone and other biosignature molecules. However, with less than $20 \%$ difference in ozone column, this has little impact on remote detection of biosignature molecules.
GCRs may also change the biologically weighted UV surface flux $W$ by up to $40 \%$. During a stellar flare, $W$ can increase much more, and reach values a factor of 3-4 higher than on Earth, or 200-300 times the quiescent level. Such values can be considered as non-critical. Also, this effect is not strongly dependent on the GCR flux, but we note that the surface UV flux on M-dwarf star planets is much more variable than what we know from Earth.

Finally, part of the energetic charged particles reach the planetary surface, where they contribute to a potentially harmful radiation background and increase the effective dose rate. This increase is only a factor of a few for the case of an Earth-like atmosphere. For planets with a thin atmosphere, however, magnetospheric shielding is important to protect the surface. This may also have important implications for studies of the possibility of life on the surface of sub-Earth-mass exoplanets close to their host star.

Overall, the potential absence of magnetic shielding against galactic cosmic rays has surprisingly little effect on the planet considered. Other effects are likely to dominate, unless the planet has a weak atmosphere and a strong magnetosphere. The case is different for stellar cosmic rays, which are analyzed in a companion article (Tabataba-Vakili et al. 2016).

Acknowledgements. This study was supported by the International Space Science Institute (ISSI) and benefited from the ISSI Team "Evolution of Exoplanet Atmospheres and their Characterisation".

\section{References}

Allen, M., Lunine, J. I., \& Yung, Y. L. 1984, J. Geophys. Res., 89, 4841

Alvarez-Muñiz, J., Engel, R., Gaisser, T. K., Ortiz, J. A., \& Stanev, T. 2002, Phys. Rev. D, 66, 033011

Atri, D., Melott, A. L., \& Thomas, B. C. 2010, J. Cosmol. Astropart. Phys., 5, 8 Atri, D., , \& Melott, A. L. 2011, Radiat. Phys. Chem., 80, 701

Atri, D., Hariharan, B., \& Grießmeier, J.-M. 2013, Astrobiology, 13, 910

Bazilevskaya, G. A., Usoskin, I. G., Flückiger, E. O., et al. 2008, Space Sci. Rev., 137,149

Belisheva, N. K., Kuzhevskii, B. M., Vashenyuk, E. V., \& Zhirov, V. K. 2005, Doklady Biochemistry and Biophysics, 402, 254

Belisheva, N. K., Kuzhevskij, B. M., Sigaeva, E. A., Panasyuk, M. I., \& Zhirov, V. K. 2006, Doklady Biochemistry and Biophysics, 407, 83

Belisheva, N. K., Lammer, H., Biernat, H. K., \& Vashenuyk, E. V. 2012 Astrophys. Space Sci. Trans., 8, 7

Buccino, A. P., Lemarchand, G. A., \& Mauas, P. J. D. 2007, Icarus, 192, 582

Cieslik, S., \& Nicolet, M. 1973, Space Sci., 21, 925

Cockell, C. S. 1999, Icarus, 141, 399

Crutzen, P. J. 1970, Q. J. R. Meteorol. Soc., 96, 320

Cuntz, M., Guinan, E. F., \& Kurucz, R. L. 2010, IAU Symp., 264, 419

Dartnell, L. R. 2011, Astrobiology, 11, 551

de Gruijl, F. R. 2000, Photocarcinogenesis: UVA vs. UVB. Method. Enzymol., 319,359

Domagal-Goldman, S. D., \& Meadows, V. S. 2010, ASP Conf. Ser., 430, 152

Dressing, C. D., \& Charbonneau, D., 2013, ApJ, 767, 95

France, K., Froning, C. S., Linsky, J. L., et al. 2013, ApJ, 763, 149

Gascón, J., Oubiña, A., Pérez-Lezaun, A., \& Urmeneta, J. 1995, Curr. Microbiol., 30, 177

Grenfell, J. L., Grießmeier, J.-M., Patzer, B., et al. 2007a, Astrobiology, 7, 208 Grenfell, J. L., Stracke, B., von Paris, P., et al. 2007b, Planet. Space Sci., 55, 661 Grenfell, J. L., Gebauer, S., von Paris, P., et al. 2011, Icarus, 211, 81

Grenfell, J. L., Grießmeier, J.-M., von Paris, P., et al. 2012, Astrobiology, 12, 1109

Grenfell, J., Gebauer, S., Godolt, M., et al. 2013, Astrobiology 13, 415

Grenfell, J. L., Gebauer, S., von Paris, P., Godolt, M., \& Rauer, H. 2014, Planet. Space Sci., 98, 66

Grießmeier, J.-M. 2014, Astrophys. Space Sci. Lib., 411, 213

Grießmeier, J.-M., Stadelmann, A., Motschmann, U., et al. 2005, Astrobiology, 5,587

Grießmeier, J.-M., Stadelmann, A., Grenfell, J. L., Lammer, H., \& Motschmann, U. 2009, Icarus, 199, 526

Grießmeier, J.-M., Tabataba-Vakili, F., Stadelmann, A., Grenfell, J. L., \& Atri, D. 2015, A\&A, 581, A44 
Gueymard, C. A. 2004, Solar Energy, 76, 423

Hauschildt, P. H., Allard, F., \& Baron, E. 1999, ApJ, 512, 377

Heath, M. J., Doyle, L. R., Joshi, M. M., \& Haberle, R. M. 1999, Origins Life Evol. Biosphere, 29, 405

Heck, D., Knapp, J., Capdevielle, J. N., Schatz, G., \& Thouw, T. 1998, (FZKA 6019), Forschungszentrum Karlsruhe, Scientific report

Heck, D., Peirog, T., \& Knapp, J. 2012, CORSIKA: An air shower simulation program, Astrophysics Source Code Library 1202.006

Hedelt, P., von Paris, P., Godolt, M., et al. 2013, A\&A, 553, A9

Horneck, G. 1995, J. Photochem. Photobiol. B: Biol., 31, 43

Hu, R., \& Seager, S. 2014, ApJ, 784, 63

Hu, R., Seager, S., \& Bains, W. 2012, ApJ, 761, 166

Hu, R., Seager, S., \& Bains, W. 2013, ApJ, 769, 6

Itikawa, Y. 2006, J. Phys. Chem. Ref. Data, 35, 31

Jackman, C. H., Frederick, J. E., \& Stolarski, R. S. 1980, J. Geophys. Res., 85, 7495

Jackman, C. H., DeLand, M. T., Labow, G. J., et al. 2005, J. Geophys. Res., 110, A09

Jones, I. T. N., \& Bayes, K. D. 1973, J. Chem. Phys., 59, 4836

Kasting, J. F., Pollack, J. B., \& Ackerman, T. P. 1984a, Icarus, 57, 335

Kasting, J. F., Pollack, J. B., \& Ackerman, T. P. 1984b, Icarus, 57, 335

Kasting, J. F., Whitmire, D. P., \& Reynolds, R. T. 1993, Icarus, 101, 108

Kerr, J. B., \& McElroy, C. T. 1993, Science, 262, 1032

Khodachenko, M. L., Ribas, I., Lammer, H., et al. 2007, Astrobiology, 7, 167

Lammer, H., Bredehöft, J. H., Coustenis, A., et al. 2009, A\&ARv, 17, 181

Lammer, H., Selsis, F., Chassefière, E., et al. 2010, Astrobiology, 10, 45

Leggett, S. K., Allard, F., Berriman, G., Dahn, C. C., \& Hauschildt, P. H. 1996, ApJS, 104, 117

Magnotta, F., \& Johnston, H. S. 1980, Geophys. Res. Lett., 7, 769

Malicet, J., Daumont, D., Charbonnier, J., et al. 1995, J. Atmos. Chem., 21, 263

Manabe, S., \& Wetherald, R. T. 1967, J. Atmos. Sci., 24, 241

Massie, S. T., \& Hunten, D. M. 1981, J. Geophys. Res., 86, 9859

Melsheimer, C., Verdes, C., Buehler, S. A., et al. 2005, Radio Sci., 40, RS1007

Mlawer, E. J., Taubman, S. J., Brown, P. D., Iacono, M. J., \& Clough, S. A. 1997, J. Geophys. Res., 102, 16663

Moortgat, G. K., \& Kudszus, E. 1978, Geophys. Res. Lett., 5, 191

O'Brien, K., Friedberg, W., Sauer, H. A., \& Smart, D. F. 1996, Environ. Int., $22, \mathrm{~S} 1$
Pavlov, A. A., \& Kasting, J. F. 2002, Astrobiology, 2, 27

Pettersen, B. R., \& Hawley, S. L. 1989, A\&A, 217, 187

Porter, H. S., Jackman, C. H., \& Green, A. E. S. 1976, J. Chem. Phys., 65, 154

Rauer, H., Gebauer, S., von Paris, P., et al. 2011, A\&A, 529, A8

Rothman, L. S., E. Gordon, I., Barbe, A., et al. 2009, J. Quant. Spectrosc. Radiat. Transfer, 110, 533

Sagan, C., Thomson, W. R., Carlson, R., Gurnett, D., \& Hord, C. 1993, Nature, 365,715

Sander, S. P., Friedl, R. R., Golden, D. M., et al. 2003, Chemical kinetics and photochemical data for use in atmospheric studies, evaluation number 14 . Tech. rep. (JPL Publication), 02

Scalo, J., Kaltenegger, L., Segura, A., et al. 2007, Astrobiology, 7, 85

Schindler, T. L., \& Kasting, J. F. 2000, Icarus, 145, 262

Schreier, F., \& Schimpf, B. 2001, in A new efficient line-by-line code for high resolution atmospheric radiation computations incl. derivatives. eds. W. L. Smith, \& Y. Timofeyev, IRS, 2000: Current problems in atmospheric radiation (A. Deepak Publishing), 381

Seager, S., Bains, W., \& Hu, R. 2013, ApJ, 775, 104

Segura, A., Krelove, K., Kasting, J. F., et al. 2003, Astrobiology, 3, 689

Segura, A., Kasting, J. F., Meadows, V., et al. 2005, Astrobiology, 5, 706

Segura, A., Meadows, V., Kasting, J. F., Crisp, D., \& Cohen, M. 2007, A\&A, 472,665

Segura, A., Walkowicz, L. M., Meadows, V., Kasting, J., \& Hawley, S. 2010, Astrobiology, 10, 751

Selsis, F., Despois, D., \& Parisot, J.-P. 2002, A\&A, 388, 985

Selsis, F., Chazelas, B., Bordé, P., et al. 2007, Icarus, 191, 453

Shea, M. A., \& Smart, D. F. 2000, Space Sci. Rev., 93, 187

Smith, D. S., Scalo, J., \& Wheeler, J. C. 2004, Icarus, 171, 229

Tabataba-Vakili, F., Grenfell, J. L., Grießmeier, J.-M., \& Rauer, H. 2016, A\&A, 585, A96

Tian, F., France, K., Linsky, J. L., Mauas, P. J. D., \& Vieytes, M. C. 2014, Earth Planet. Sci. Lett., 385, 22

Toon, O. B., McKay, C. P., Ackerman, T. P., \& Santhanam, K. 1989, J. Geophys. Res., 94, 16287

Vardavas, I. M., \& Carver, J. H. 1984, Planet. Space Sci., 32, 1307

von Paris, P., Cabrera, J., Godolt, M., et al. 2011, A\&A, 534, A26

von Paris, P., Hedelt, P., Selsis, F., Schreier, F., \& Trautmann, T. 2013, A\&A 551, A120

Yao, F., Wilson, I., \& Johnston, H. 1982, J. Phys. Chem., 86, 3611 